\title{
Dynamic compression of foam supported plates impacted by high velocity soil
}

\author{
T. Liu*, H.N.G. Wadley ${ }^{\S}$ and V.S. Deshpande* \\ *Department of Engineering, University of Cambridge, Trumpington Street, \\ Cambridge CB2 1PZ, UK. \\ ${ }^{\S}$ Department of Material Science \& Engineering, \\ School of Engineering and Applied Science, University of Virginia \\ Charlottesville, VA 22903, USA.
}

\begin{abstract}
The response of back-supported buffer plates comprising a solid face sheet and foam core backing impacted by a column of high velocity particles (sand slug) is investigated via a lumped parameter model and coupled discrete/continuum simulations. The buffer plate is either resting (unattached) or attached to a rigid stationary foundation. The lumped parameter model is used to construct maps of the regimes of behaviour with axes of the ratio of the height of the sand slug to core thickness and the normalised core strength. Four regimes of behaviour are identified based on whether the core compression ends prior to the densification of the sand slug or vice versa. Coupled discrete/continuum simulations are also reported and compared with the lumped parameter model. While the model predicted regimes of behaviour are in excellent agreement with numerical simulations, the lumped parameter model is unable to predict the momentum transmitted to the supports as it neglects the role of elasticity in both the buffer plate and the sand slug. The numerical calculations show that the momentum transfer is minimised for intermediate values of the core strength when the so-called "soft-catch" mechanism is at play. In this regime the bounce-back of the sand slug is minimised which reduces the momentum transfer. For high values of the core strength, the response of the buffer plate resembles a rigid plate with nearly no impulse mitigation while at low values of core strength, a slap event occurs when the face sheet impinges against the foundation due to full densification of the foam core. This slap event results in a significant enhancement of the momentum transfer to the foundation. The results demonstrate that appropriately designed buffer plates have potential as impulse mitigators in landmine loading situations.
\end{abstract}

Keywords: fluid-structure interaction, discrete/continuum coupling, dynamic loading.

Submitted to International Journal of Impact Engineering, August 2012 


\section{Introduction}

The air and water blast resistance of structures has recently received increased attention with the overall aim of designing lightweight, blast resistant structures. Several recent theoretical studies have shown that sandwich structures subjected to water blast outperform monolithic structures of equal mass, see for example Fleck and Deshpande [1] and Xue and Hutchinson [2]. Experiments reported by Wadley et al [3] and Wei et al [4] have confirmed these predictions. The enhanced performance is mainly due to fluid-structure interaction effects such that a smaller fraction of the impulse is transmitted into sandwich structures compared to their monolithic counterparts. By contrast, under air blast, sandwich structures provide smaller benefits over monolithic structures as fluid-structure interaction effects are more difficult to exploit [5]. The extension of these ideas to the design of structures against soil impact from say a landmine explosion (see Fig. 1) requires better insight into the dynamic interaction of soil with structures; the development of such a fundamental understanding is the focus of this article.

Significant understanding of fluid-structure interactions effects for underwater and air blast loading has been obtained via one-dimensional calculations. For example, following the classical work of Taylor [6] which quantified the underwater fluidstructure interaction effects for a free-standing rigid plate, Deshpande and Fleck [7] extended these ideas to sandwich plates. Deshpande and Fleck [7] predicted significant reductions in the momentum transfer to sandwich plates compared to monolithic plates of equal mass. These predictions were verified via simulated underwater blast experiments $[8,9]$ that involved the detonation of an underwater charge. Analogous predictions were also reported for air explosions by Kambouchev et al. [5] and Hutchinson [10] and subsequently investigated experimentally in [11,12]. Given the insight that has been gleaned from these studies, we develop here an analogous model to investigate the fluid-structure interaction between an impacting column of particles (representing the ejecta of a landmine) and a back-supported buffer plate.

A time sequence of the ejecta from a buried explosion is shown in Fig. 2a. It can be seen that the initially ejected soil forms a circular column with an approximately planar leading edge. Wadley and co-workers ${ }^{1}$ have recently investigated the response of back-supported sandwich structures impacted by model explosively accelerated "planar leading edge" sand columns. The experimental set-up used in their experiments is sketched in Fig. $2 \mathrm{~b}$ and involves: (i) a sandwich panel bolted to a vertical pendulum at a stand-off $S$ from the ground and (ii) a planar explosive sheet buried a depth $h$ under a granular medium comprising water saturated mono-sized glass spheres. Detonation of the explosive results in a column of granular media with a planar front rising and impacting the sandwich panel. The experiments measure the permanent core compression of the sandwich panel and the momentum transferred by the panel into the underlying foundation via the rise of the vertical pendulum.

In order to gain fundamental insight into these experiments we have devised the onedimensional model problem as sketched in Fig. 3a. In this model problem, a column of particles, all travelling at the same velocity, impact a back-supported buffer plate. The column of particles is constrained from expanding laterally thereby making this a

\footnotetext{
${ }^{1}$ Unpublished work.
} 
quasi one-dimensional problem. The model problem of course neglects a range of complicated phenomena that are at play in the experiments including: (i) edge effects due to the lateral flow of the granular media; (ii) interaction of the high velocity granular media with the surrounding air and (iii) velocity and density gradients within the impacting sand column. However, we restrict attention to this simplified model problem in order to gain an understanding of the key physical phenomena at play.

Numerical methods to solve fluid/structure interaction problems have typically focussed on a coupled Eulerian/Lagrangian approach wherein the granular media is represented within an Eulerian setting and the structure within a Lagrangian setting. However, the successful implementation of this coupled Eulerian-Lagrangian computational framework has been elusive due to computational problems associated with the analysis of low density particle sprays; see for example Wang et al. [13] for a discussion of these numerical issues. An alternative modelling strategy has recently been employed by Borvik et al. [14] and Pingle et al. [15]. In this approach the low density soil is treated as an aggregate of particles with the contact law between particles dictating the overall aggregate behaviour. On the other hand, the structure is treated as a continuum within a Lagrangian finite element (FE) setting. The media described by the discrete and continuum approaches are coupled via an explicit scheme. This approach has several advantages: (i) there is no need to make a-priori assumptions about the constitutive response of the aggregate (this becomes an outcome of the simulations), (ii) it provides a fundamental tool to study the essential physics of the sand-structure interaction and (iii) given that the sand is represented by a discrete set of particles we do not face the usual numerical problems associated with solving the equations associated with the equivalent continuum descriptions. Pingle et al. [15] have recently investigated the response of a rigid target to impact by a column of particles while in a parallel study, Borvik et al. [14] analysed the response of monolithic plates to a spherically-expanding sand shell and compared their predictions with measurements. More recently Liu et al. [16,17] have used this technique to investigate the response of clamped beams and plates subject to impact by a column of particles. In this investigation we employ the coupled discrete/continuum approach of Liu et al. [16] to investigate the problem described above.

\subsection{Approach and scope}

Our aim is to develop overall fundamental understanding of the fluid-structure interaction between columns of granular media and back-supported buffer plates with foam cores. We first develop a semi-analytical lumped parameter for the problem and use the model to construct mechanism regime maps. Next we describe the numerical methods developed for the discrete particle calculations, and the Lagrangian finite element (FE) simulations. The numerical results are then presented and comparisons made with the lumped parameter model in terms regimes of behaviour, core compression and momentum transfer. By using both approaches we are able to identify the physical phenomena governing momentum transfer and the effect of a foam backing on this process.

\section{Regimes of behaviour}

In order to develop a fundamental understanding of the response of the backsupported buffer plate investigated by Wadley and co-workers (Fig. 2b); we consider 
the idealised boundary value problem sketched in Fig. 3a. Consider a compound buffer plate of width $W$ with a front face sheet of thickness $h$ with a material density $\rho_{f}$ and a cellular core of thickness $c$ and and density $\rho_{c}$, as shown in Fig. $3 \mathrm{~b}$. The plate rests on a rigid foundation. We consider two limiting attachment conditions of the buffer plate:

(i) The plate rests on but is not attached to the rigid foundation so that only compressive stresses may be transmitted into the foundation. We shall subsequently refer to this case as the unattached buffer plate.

(ii) The buffer plate is bonded to the rigid foundation so that tensile stresses may be transmitted into the foundation with no failure of the interface between the buffer and foundation. We shall subsequently refer to this as the attached buffer plate case.

A sand slug of height $H$ impacts the front face of the buffer plate. The slug has a uniform spatial distribution of sand particles all travelling with an initial velocity $v_{o}$ in the negative $x_{2}$ direction. The sand slug has an initial relative density $\bar{\rho}$ and comprises particles made from a solid with density $\rho_{S}$. As sketched in Fig. 3a we constrain the slug from spreading laterally and it impacts the sandwich plate over its entire span at time $t=0$. The constraint against lateral spreading is representative of the situation near the centre of a sandwich panel loaded by a sand spray with zero obliquity. In such a situation, the sand at the periphery constrains the lateral flow of the sand at the centre. With the sandwich properties assumed to be invariant in the $x_{1}$ direction, the boundary value problem reduces to a quasi one-dimensional (1D) problem for panel widths $W \gg D$, where $D$ is the representative size of the sand particles.

\section{$2.1 \quad$ A lumped parameter model}

Before presenting full numerical simulations for the response of the buffer plates impacted by the sand slug as illustrated in Fig. 3a, we develop a simplified semianalytical approach to understand the response over a wide range of parameters. This semi-analytical model is analogous to the one-dimensional "lumped parameter" model developed by Deshpande and Fleck [7] for the water blast response of freestanding sandwich plates and is based on idealised one-dimensional responses for the sandwich core, face sheets and the impacting sand.

The front face of the buffer plate is treated as rigid with an areal mass $m_{f} \equiv \rho_{f} h$ while the core is modelled as a rigid ideally-plastic foam-like solid. It compresses at a constant strength $\sigma_{c}$ with no lateral expansion up to a densification strain $\varepsilon_{D}$ beyond which it is rigid; see Fig. 4a. This type of constitutive law is representative of foams $[18,19]$ and other stacked, periodic cellular cores such as the prismatic diamond and stacked pyramidal cores [20]. It is commonly referred to as the rigid, perfectly plastic locking (rppl) solid. The rppl solid was first introduced by Reid and Peng [21] and is properly interpreted as the limit of an elastic-hardening solid with elastic modulus tending to infinity and the plastic modulus tending to zero. The neglect of elastic deformation of the core is justified when the time for multiple elastic wave reflections in the core is much less that the time for plastic wave propagation through the core. This is expected to be true for most cellular solids since the elastic wave speeds in these solids are much higher than the plastic wave speed. Similarly, the assumption 
of a rigid face sheet is acceptable when the transit time for multiple elastic wave reflections in the face sheets is much less than the time for wave propagation in the core. This is an acceptable approximation in most cellular core sandwich structures where the faces are much thinner than the core.

Recall that the impacting sand slug comprises a spatially uniform relative density $\bar{\rho}$ of particles all travelling at an initial velocity $v_{o}$ in the negative $x_{2}$ direction. Since the lateral expansion of this slug is constrained, the notional quasi-static compressive stress versus nominal compressive strain response of the slug can be idealised by the curve sketched in Fig. 4b. In this case the slug compresses up to its densification strain $\varepsilon_{S}$ at zero stress whereupon the particles come into contact. In this simplified analysis, we specify that the slug behaves in a rigid manner beyond this densification strain. Thus, the form of the quasi-static responses of the sand slug and the core are similar with the main difference being that prior to densification, the core has a strength $\sigma_{c}$ while the sand slug has no strength prior to its densification.

The key simplification in this model is that elasticity is neglected both in the buffer plate and the sand slug. This assumption implies that the predictions of this model for both the attached and unattached boundary conditions mentioned above are identical. The numerical calculations discussed in Section 3 include the effects of elasticity in both the buffer plate and the sand slug; these numerical calculations will be subsequently employed to understand the limitations of this lumped parameter model. We now proceed to analyse this simplified one-dimensional problem.

At $t=0$ the buffer plate is stationary while the sand slug has a uniform velocity $v_{o}$ in the negative $x_{2}$ direction. Two distinct cases exist:

Case (I): a plastic shock wave propagates into the core after the impact of the sand slug; and

Case (II): no plastic shock wave is initiated in the core.

We shall consider each case in turn and then derive conditions for their applicability.

\subsubsection{Case (I): A plastic shock wave propagates into the core}

After impact, a plastic shock wave is initiated both in the core and the sand slug. These plastic shocks travel at Lagrangian speeds $c_{f}$ and $c_{s}$ in the negative and positive $x_{2}$ directions within the foam core and the sand slug, respectively as indicated in Fig. 4c. After time $t$, these shock fronts have travelled a distance $s_{f}$ and $s_{s}$ (as measured in the undeformed configuration) within the core and sand slug, respectively. The plastic shocks compress the foam core to its densification strain $\varepsilon_{D}$ while the sand is also densified by the shock to a strain $\varepsilon_{S}$. The rigid nature of the responses of the core and the sand slug after densification implies that at time $t$ the portions of the sand slug and core that are engulfed by the plastic shock as well as the front face sheet have a common velocity $v$; the remainder of the sand slug is undeformed and has its initial velocity $v_{o}$ in the negative $x_{2}$ direction while the core downstream from shock is also undeformed and at rest. 
Let the interfacial pressure between the sand slug and the front face of the buffer plate be $p$ while $\sigma_{f}$ and $\sigma_{S}$ are the stresses immediately downstream from the shocks in the core and sand slug, respectively. Recalling that the densified core and sand slugs behave as rigid bodies, the equations of motion for the common velocity $v$ are given as

$$
\left(m_{f}+\rho_{c} s_{f}\right) \dot{v}=p-\sigma_{f}
$$

and

$$
\bar{\rho} \rho_{S} S_{S} \dot{v}=\sigma_{S}-p
$$

where the overdot denotes differentiation with respect to time. Conservation of momentum across the plastic shocks in the core and the sand slug dictate that the downstream stresses $\sigma_{f}$ and $\sigma_{S}$ are related to the shock speeds via the relations

and

$$
\sigma_{f}=\sigma_{c}+\rho_{c} c_{f} v
$$

$$
\sigma_{S}=\bar{\rho} \rho_{S} c_{S}\left(v_{o}-v\right)
$$

respectively. Mass conservation across the shocks gives the plastic shock velocities in the core and the sand slug as

and

$$
c_{f} \equiv \dot{s}_{f}=\frac{v}{\varepsilon_{D}}
$$

$$
c_{S} \equiv \dot{s}_{S}=\frac{v_{o}-v}{\varepsilon_{S}},
$$

respectively. Equations (2.1)-(2.6) are a set of coupled ordinary differential equations (ODEs) for the quantities $v, s_{f}$ and $s_{S}$ that can be solved numerically with initial conditions $v=s_{f}=s_{S}=0$ in order to get the responses of both the buffer plate and sand slug.

We proceed to analyse this further by first presenting the equations in nondimensional form. Introduce the non-dimensional loading parameters

$$
\bar{\sigma}_{c} \equiv \frac{\sigma_{c} \varepsilon_{S}}{\bar{\rho} \rho_{S} v_{o}^{2}} \quad \text { and } \quad \psi \equiv \frac{\bar{\rho} \rho_{S} H}{m_{f}}
$$

where $\bar{\sigma}_{c}$ is the ratio of the strength of the core to the pressure exerted by the sand on a rigid-stationary structure [15] while $\psi$ is analogous to the Taylor [6] fluid-structure interaction parameter in water blast and is the ratio of the areal mass of the sand to the areal mass of the face sheet of the buffer plate. The buffer plate is characterised by the non-dimensional groups

$$
\bar{H} \equiv \frac{H}{c} \quad \text { and } \quad \bar{m} \equiv \frac{m_{f}}{\rho_{c} c}
$$

Introducing a non-dimensional time $\bar{t} \equiv t v_{o} / H$ and a non-dimensional velocity $\bar{v} \equiv v / v_{o}$ we can simplify and re-write Eqs. (2.1) to (2.6) in non-dimensional form as 


$$
\begin{gathered}
\dot{\bar{v}}=\frac{\psi \bar{m} \varepsilon_{D}(1-\bar{v})^{2}-\bar{H} \varepsilon_{S} \bar{v}^{2}-\bar{\sigma} \psi \bar{m} \varepsilon_{D}}{\bar{H} \varepsilon_{D} \varepsilon_{S} \bar{s}_{f}+\psi \bar{m} \varepsilon_{D} \varepsilon_{S} \bar{s}_{S}+\bar{m} \varepsilon_{D} \varepsilon_{S}} \\
\dot{\overline{s_{f}}}=\frac{\bar{v}}{\varepsilon_{D}}
\end{gathered}
$$

and

$$
\dot{\overline{S_{S}}}=\frac{1-\bar{v}}{\varepsilon_{S}}
$$

where the overdot now denotes differentiation with respect to the non-dimensional time $\bar{t}$ while $\bar{s}_{f} \equiv s_{f} / c$ and $\bar{s}_{S} \equiv s_{S} / H$. These three coupled ODEs with initial conditions $\bar{v}=\bar{s}_{f}=\bar{s}_{S}=0$ at time $\bar{t}=0$ are solved numerically ${ }^{2}$ to obtain the average through-thickness core compression $\varepsilon_{c} \equiv \bar{s}_{f} \varepsilon_{D}$ as a function of $\bar{t}$. The equations presented in this section are valid for a core that is sufficiently weak to initiate a plastic shock wave. Employing the initial condition that $\dot{\bar{v}}>0$ at $\bar{t}=0^{+}$we see from Eq. (2.9) that the condition for a plastic shock wave initiation is $\bar{\sigma}_{c}<1$.

The above set of equations is valid if $\bar{\sigma}_{c}<1$ and until one of the following events occur:

Event (i): The plastic shock wave in the core reaches the rear end of the core (i.e. $\left.\bar{s}_{f}=1\right)$ resulting in full densification of the core.

Event (ii): The plastic shock within the sand slug reaches the top free surface of the slug (i.e. $\bar{s}_{S}=1$ ) resulting in the complete densification of the sand slug.

We note in passing that with $\bar{\sigma}_{c}<1$, plastic shocks cannot arrest in either the core or the sand slug prior to the occurrence of one of the two events above. This is rationalised as follows:

(a) The condition for the shock to arrest within the core is $\dot{\bar{v}}=\bar{v}=0$. Substituting $\bar{v}=0$ in Eq. (2.9) and recalling that $\bar{\sigma}_{c}<1$ we observe that $\dot{\bar{v}}>0$ when $\bar{v}=0$. Thus, the shock cannot arrest within the core while Eqs. (2.9) to (2.11) are valid.

(b) The conditions that must be met for the shock to arrest within the sand slug are $\bar{v}=1$ with $\dot{\bar{v}}=0$. Substituting $\bar{v}=1$ in Eq. (2.9), we observe that $\dot{\bar{v}}<0$ indicating that the shock cannot arrest within the sand slug while Eqs. (2.9) to (2.11) are valid.

We shall now consider each of the above two events in turn.

\subsubsection{Event (i): Governing equations after full densification of the core}

Consider Event (i) described above such that a plastic shock reaches the rear end of the core at time $\bar{t}=\bar{t}_{0}$ when $\bar{s}_{S}=\bar{s}_{S 0}<1$, i.e. the sand slug has not yet fully densified. With elasticity in the core and the face sheet neglected in this model we assume that the front face sheet is instantaneously brought to rest at time $\bar{t}=\bar{t}_{0}$ and the buffer plate now behaves as a rigid body, i.e. $v=0$ with only plastic shock wave propagation within the sand slug. With $v=0$, Eqs. (2.2), (2.4) and (2.6) reduce to

\footnotetext{
${ }^{2}$ The MATLAB function ode23 based on an automatic step-size Runga-Kutta-Fehlberg integration method was employed.
} 


$$
p=\sigma_{S}=\bar{\rho} \rho_{S} \frac{v_{o}^{2}}{\varepsilon_{S}}
$$

This equation is valid until the sand slug fully densifies at time $\bar{t}=\bar{t}_{0}+H\left(1-\bar{s}_{S 0}\right) / v_{o}$ whereupon the pressure $p=0$.

\subsubsection{Event (ii): Governing equations after full densification of the sand slug.}

Consider Event (ii) described above such that complete densification of the sand slug occurs at time $\bar{t}=\bar{t}_{1}$ while the plastic shock wave is still propagating within the core. At time $\overline{t_{1}}$ the shock wave within the core is located at $\bar{s}_{f}=\bar{s}_{f 1}<1$ and the common velocity upstream from the shock is $\bar{v}_{1}$. For times $\bar{t}>\bar{t}_{1}$, we assume that the densified sand slug behaves as a rigid mass attached to the front face of the buffer plate. The equations governing the response of the buffer plate as given in Section 2.1.1 can then be simplified by not accounting for plastic shock wave propagation within the sand slug, i.e. Eqs. (2.1), (2.3) and (2.5) remain unchanged and Eq. (2.2) reduces to

$$
\bar{\rho} \rho_{S} H \dot{v}=-p
$$

with $\sigma_{S}=c_{S}=0$. In non-dimensional form the overall governing equations for the response of the buffer plate are then give as

$$
\left(\bar{m}+\psi \bar{m}+\bar{H} \bar{s}_{f}\right) \dot{\bar{v}}=-\left(\frac{\bar{\sigma}_{c} \bar{m} \psi}{\varepsilon_{S}}+\dot{\overline{s_{f}}} \bar{H} \bar{v}\right)
$$

and

$$
\dot{\bar{S}}_{f}=\frac{\bar{v}}{\varepsilon_{D}}
$$

with initial conditions $\bar{s}_{f}=\bar{s}_{f 1}$ and $\bar{v}=\bar{v}_{1}$ at time $\bar{t}_{1}$ when the sand slug was fully densified. Here again the overdot denotes differentiation with respect to the nondimensional time $\bar{t}$. Equations (2.14) and (2.15) are valid until one of the two following events occur:

Event $(i i-a)$ : The plastic shock arrests within the core, i.e. $\dot{\bar{s}}_{f}=0$ with ${\overline{s_{f}}}_{f}<$. At this instant the deformation ends with all components having been brought to rest.

Event (ii-b): The plastic shock wave impinges on the foundation, i.e. $\bar{s}_{f}=1$ with $\dot{\bar{s}}_{f}>0$. In line with the assumptions detailed in Section 2.1.2 we neglect any subsequent rebounding of the buffer plate and assume all components are instantly brought to rest when the plastic shock wave in the core reaches the rear end of the core.

\subsubsection{Case (II): No plastic shock wave is initiated in the core}

When $\bar{\sigma}_{c}<1$, no plastic shock wave is initiated in the core and the sand slug exerts the pressure given by Eq. (2.12) from $\bar{t}=0$ until densification of the slug at time $\bar{t}=1$, whereupon the pressure drops to zero.

\subsection{Regime maps}

The main application of the lumped parameter model is to predict the regimes of behaviour for the response of buffer plates impacted by sand slugs as well as the core 
compression. The analysis of Section 2.1 suggests four regimes of behaviour based on the sequence of the compression of the core and the sand slug:

(i) Regime I: Densification of the core followed by densification of the sand slug.

(ii) Regime II: Densification of the sand slug followed by densification of the core.

(iii)Regime III: Densification of the sand slug followed by end of core compression without complete densification of the core, (i.e. the plastic shock wave arrests within the core and does not reach the rear face).

(iv) Regime IV: Densification of the sand slug with no shock wave initiated in the core.

The final (or maximum) average through-thickness core compressive strain in the core $\varepsilon_{c}^{\max }=\varepsilon_{D}$ in regimes I and II while $\varepsilon_{c}^{\max }=0$ in regime IV. The maximum core compression varies over the range $0 \leq \varepsilon_{c}^{\max } \leq \varepsilon_{D}$ in regime III.

Consider a buffer plate with $\bar{m}=1$, impacted by a sand slug with relative density $\bar{\rho}=0.2$. Maps illustrating these regimes of behaviour with axes of non-dimensional core strength $\bar{\sigma}_{c}$ and slug height $\bar{H}$ are plotted in Figs. 5a and 5b for the choices of the fluid-structure interaction parameter $\psi=0.7$ and 10 , respectively. In plotting these maps we assumed that the core densification strain $\varepsilon_{D}=0.8$ while the densification strain for the sand is given as

$$
\varepsilon_{s}=1-\frac{\bar{\rho}}{\bar{\rho}_{\max }}
$$

where $\bar{\rho}_{\max }$ is the maximum packing density of the sand particles. Here we take $\bar{\rho}_{\max }=0.9$ so as to match the maximum packing density of an array of identical discs or cylinders; such discs are used in the numerical calculations reported in Section 3. The main effect of increasing the value of $\psi$ from 0.7 to 10 is the area of the map occupied by regimes II and III shrinks with regime I now dominating the map. For a fixed face sheet mass per unit area, a higher value of $\psi$ implies that the amount of sand impacting the plate increases, i.e. the length of the sand slug increases. This longer sand slug takes more time to fully densify and thus core compression is completed before densification of the sand slug over a larger portion of the map.

In order to illustrate the core compression behaviour predicted by the lumped parameter model, we now consider the map in Fig. 5a and select four representative geometries in regimes I to IV, we shall refer to these as geometries G-I, G-II, G-III and G-IV, respectively. These geometries are marked by open circles in Fig. 5a. The predicted evolution of the normalised average through-thickness core compression $\varepsilon_{c} / \varepsilon_{D}$ with normalised time $\bar{t}$ is plotted in Fig. 6a. Geometries G-II and G-III have approximately equal initial compression rates but geometry G-III attains a maximum value $\varepsilon_{c}^{\max } / \varepsilon_{D} \approx 0.35$ while core compression in geometry G-II continues until there is complete densification of the core with $\varepsilon_{c}^{\max } / \varepsilon_{D}=1$. The higher value of $\bar{H}$ for geometry G-I means that G-I undergoes a higher initial compression rate with core compression ending when $\varepsilon_{c}^{\max } / \varepsilon_{D}=1$. Contours of the normalised core compression $\varepsilon_{c}^{\max } / \varepsilon_{D}$ are included in regime III in Fig. 5(recall that $\varepsilon_{c}^{\max } / \varepsilon_{D}=1$ in regimes I and II and $\varepsilon_{c}^{\max } / \varepsilon_{D}=0$ in regime IV) and indicate that $\varepsilon_{c}^{\max }$ varies 
smoothly from $\varepsilon_{c}^{\max }=0$ at the regime III and IV boundary to $\varepsilon_{c}^{\max } / \varepsilon_{D}=1$ at the boundaries between regimes III and regimes I and II.

In order to illustrate the effect of core strength on core compression more clearly, we plot the core compression $\varepsilon_{c}^{\max } / \varepsilon_{D}$ as a function of $\bar{\sigma}_{c}$ by taking three slices through the map in Fig. 5a at $\bar{H}=0.2,1$ and 10. These slices are included in Fig. 6b and indicate that while the transition from no compression $\left(\varepsilon_{c}^{\max } / \varepsilon_{D}=0\right)$ to full core compression $\left(\varepsilon_{c}^{\max } / \varepsilon_{D}=1\right.$ ) occurs rather abruptly when $\bar{\sigma}_{c}$ decreases from 1 to 0.8 for the $\bar{H}=10$ case. By contrast, the transition is much more gradual for the lower values of $\bar{H}$. This arises becasue intermediate values of core compression occur only in Regime III and this regime is expanded by decreasing $\bar{H}$; see Fig. 5a.

\subsection{Momentum transfer}

With elasticity in both the buffer plate and the sand slug neglected in the lumped parameter model we assume that no bounce-back of the buffer plate or sand slug occurs even for the unattached buffer plates. Thus, the model predicts the total areal momentum $I_{t}^{\text {total }}$ transferred to the foundation equals the momentum $I_{o}=\bar{\rho} \rho_{S} H v_{o}$ of the incoming sand slug, i.e. $I_{t}^{\text {total }} / I_{o}=1$ in all cases. This is a major drawback of the lumped parameter model and we now develop coupled discrete/continuum simulations where elasticity effects in both the buffer plate and sand slug are included.

\section{Coupled discrete particle/finite element calculations}

The simplified lumped parameter model presented in Section 2 gives a broad overview of the response of back-supported buffer plates and makes predictions of the core compression. However, there are a number of simplifying assumptions in the model which include:

(i) Elasticity of the face sheet, core and densified sand aggregate is neglected. This implies that the model predicts that the momentum transmitted into the foundation is equal to the initial momentum of the sand slug over all values of the parameters in the problem.

(ii) The model cannot differentiate between the attached and unattached boundary conditions of the buffer plate.

In this section we present two-dimensional (2D) calculations where the sand slug is modelled by a large number of discrete identical cylindrical particles, while the buffer plate is modelled as a $2 \mathrm{D}$ continuum with an elastic face sheet and a foam core. These 2D calculations have the required level of sophistication to account for bounceback effects of the buffer plate and the sand slug and thus provide an indication of the fidelity of the lumped parameter model and the regimes within which that simplified model provides accurate predictions.

\subsection{Summary of the numerical procedure}

The sand slug was modelled as an aggregate of 2D discrete identical cylindrical particles (or discs) using the GRANULAR package in the multi-purpose molecular dynamics code LAMMPS [22] while the buffer plate was modelled using a 2D finite 
strain Lagrangian finite element framework. We shall briefly describe each of these methodologies followed by details of the coupling of these two techniques.

Discrete particle calculations: The granular medium was modelled by a random array of 2D cylindrical particles, each of diameter $D$ lying in the $x_{1}-x_{2}$ plane (unit thickness in the $x_{3}$-direction); see Fig. 3a. The granular package in LAMMPS is based on the soft-particle contact model as introduced by Cundall and Strack [23] and extended to large scale simulations by Campbell and co-workers [24,25]. This softparticle contact model idealises the deformation of two contacting particles, each of mass $m_{p}$, as depicted in Fig. 7. The contact law comprises:

(i) a linear spring $K_{n}$ and a linear dashpot of damping constant $\gamma_{n}$ in parallel, governing the normal motion and

(ii) a linear spring $K_{s}$ and a Coulomb friction element of coefficient $\mu$, in series, governing the tangential motion during contact.

The contact forces in the normal and tangential directions are now specified as follows. Write $r$ as the separation of particle centres and $\delta_{n}=r-D$ as the interpenetration. During active contact $\left(\delta_{n}<0\right)$, the normal force is given by

$$
F_{n}=K_{n} \delta_{n}+m_{\text {eff }} \gamma_{n} \dot{\delta}_{n}
$$

where $m_{\text {eff }}$ is the effective or reduced mass of the two contacting bodies. We take $m_{\text {eff }}=m_{p} / 2$ for impacts between particles, and $m_{\text {eff }}=m_{p}$ for impacts between a particle and the plate.

The tangential force $F_{s}$ only exists during active contact, and opposes sliding. It is limited in magnitude to $\left|F_{s}\right|<\mu\left|F_{n}\right|$ as follows. Define $\dot{\delta}_{s}$ as the tangential displacement rate between the contacting particles. Then, $F_{s}$ is given by an "elasticplastic" relation of Coulomb type;

$$
\dot{F}_{s}= \begin{cases}K_{s} \dot{\delta}_{s} & \text { if }\left|F_{s}\right|<\mu\left|F_{n}\right| \text { or } F_{s} \dot{\delta}_{s}<0 \\ 0 & \text { otherwise }\end{cases}
$$

The value of damping constant $\gamma_{n}$ dictates the loss of energy during normal collision and is directly related to the coefficient of restitution $e$ according to

$$
e=\exp \left[-\pi\left(\frac{8 K_{n}}{\gamma_{n}^{2} m_{p}}-1\right)^{-1 / 2}\right]
$$

The collision time for individual binary collisions $t_{e}$ follows from (3.1) as

$$
t_{e}=-\frac{2 \ln (e)}{\gamma_{n}}
$$

Thus, in the limit of plastic collisions with $e \rightarrow 0$, the contact time is $t_{e} \rightarrow \infty$.

The calculations with the above contact model were performed using the GRANULAR package within the molecular dynamics code LAMMPS. The 
translational and rotational motions of the particles were obtained by integration of the accelerations using a Verlet time-integration scheme (i.e. Newmark-Beta with $\beta=0)$. The time-step within LAMMPS was typically taken to be $t_{e} / 10$ in order to ensure accurate integration of the contact relations, Eqs. (3.1)-(3.2), and this value was also used to define the time steps for the finite element calculations, as described below.

$2 D$ finite element calculations: The 2D plane strain calculations were performed using an updated Lagrangian finite element (FE) scheme with the current configuration at time $t$ serving as the reference. The co-ordinate $x_{i}$ denotes the position of a material point in the current configuration with respect to a fixed Cartesian frame, and $v_{i}$ is the velocity of that material point. For the plane strain problem under consideration, the principle of virtual power (neglecting effects of gravity) for a volume $V$ and surface $S$ is written in the form

$$
\int_{V} \sigma_{i j} \delta \dot{\varepsilon}_{i j} d V=\int_{S_{T}} T_{i} \delta v_{i} d S-\int_{V} \rho \delta \dot{v}_{i} \delta v_{i} d V
$$

where $\sigma_{i j}$ is the Cauchy stress, $\dot{\varepsilon}_{i j} \equiv 0.5\left(v_{i, j}+v_{j, i}\right)$ is the strain rate, $T_{i}$ the tractions on the surface $S_{T} \in S$ due to the impacts of the particles while $\rho$ is the material density in the current configuration. The symbol $\delta$ denotes arbitrary virtual variations in the respectively quantities. A finite element discretisation based on linear, plane strain three node triangular elements (i.e. constant strain triangles) is employed. When the finite element discretisation of the displacement field is substituted into the principle of virtual power (3.5) and the integrations are carried out, the discretised equations of motion are obtained as

$$
\mathbf{M} \frac{\partial^{2} \mathbf{U}}{\partial t^{2}}=\mathbf{F}
$$

where $\mathbf{U}$ is the vector of nodal displacements, $\mathbf{M}$ is the mass matrix and $\mathbf{F}$ is the nodal force vector. An explicit time integration scheme based on the Newmark $\beta$ method with $\beta=0$ was used to integrate Eq. (3.6) to obtain the nodal velocities and the nodal displacements. A lumped mass matrix is used in (3.6) instead of a consistent mass matrix, since this is preferable for explicit time integration procedures, for both accuracy and computational efficiency.

Coupling of the discrete and finite element calculations: At time $t$, contact between the particles and the plate in its current configuration was detected. The displacement $\delta_{n}$ is defined as the $\delta_{n}=r-D / 2$, where $r$ is the distance between the particle centre and the contact point on the plate. The rates $\dot{\delta}_{n}$ and $\dot{\delta}_{s}$ are calculated as the relative velocities of the particle and the point of contact on the plate surface. (The velocity of any point on the plate is calculated by interpolating the nodal velocities using the shape functions of the constant strain triangle elements.) The normal and tangential contact forces are then calculated using Eqs. (3.1) - (3.2) and transformed to the global co-ordinate system. This gives a vector of equivalent nodal forces for each element on the surface of the plate. These vectors of elemental nodal forces are inserted into the vector of global nodal forces $\mathbf{F}$ in Eq. (3.5). Subsequently, the discrete and finite element equations were integrated as described above and the new 
positions and velocities of the particles in the discrete calculations and material points in the finite element calculations determined at time $t+\Delta t$.

\subsection{Boundary value problem and material properties}

In the numerical calculations we analysed the impact of the sand slug on the buffer plate comprising an elastic face sheet and a foam core as well as a reference elastic block as sketched in Figs. $3 \mathrm{~b}$ and $3 \mathrm{c}$, respectively. The calculations are carried out in a $2 \mathrm{D}$ setting with deformations of the plates occurring in the $x_{1}-x_{2}$ plane shown and unit thickness in the $x_{3}$-direction.

Two boundary conditions are considered for both the elastic block and the buffer plate: (i) In the unattached case the structures (i.e. the elastic blocks or buffer plates) were assumed to rest on the rigid foundation with no penetration permitted into the foundation. However, the structures could loose contact with the foundation as the interface between the structures and the foundation was assumed to sustain no tensile stresses.

(ii) In the attached case the bottom surface of the elastic block as well as the buffer plate were fixed to the foundation, i.e. displacements $u_{2}=0$ in the $x_{2}$ direction, were specified over the bottom surfaces of the structures.

The elastic block and buffer plate of width $W$ (unit thickness in the $x_{3}$ direction) are impacted by a sand slug also of width $W$ and height $H$ as shown in Fig. 3a. The slug comprises an initial spatially uniform relative density $\bar{\rho}$ of cylindrical particles and is constrained against lateral expansion by two rigid walls located at $x_{1}=(0, W)$ as shown in Fig. 3a. Also consistent with this no lateral spreading constraint of the sand slug, we also prevent lateral expansion of the plates by imposing the displacement boundary condition $u_{1}=0$ on $x_{1}=(0, W)$ for both the plate types.

Material properties: The sand slug comprised identical cylindrical particles of diameter $D=200 \mu \mathrm{m}$ and unit thickness in the $x_{3}$ all travelling with an initial velocity $v_{o}=400 \mathrm{~ms}^{-1}$ in the negative $x_{2}$ direction. The sand particles are made from a solid material of density $\rho_{s}=2700 \mathrm{~kg} \mathrm{~m}^{-3}$. The normal stiffness between the particles was taken to be $K_{n}=7300 \mathrm{kNm}^{-1}$ and the co-efficient of restitution for both impacts between the particles and the particles and the plates was taken to be $e=0.7$ (i.e. the value of $\gamma_{n}$ for inter-particle contacts was twice that for impacts between the particles and the walls). Following Silbert et al. [26] we fixed the ratio $K_{s} / K_{n}=2 / 7$ and the reference value of the friction co-efficient was assumed to be $\mu=0.7$. We note in passing that similar to the findings of Pingle et al. [15] for the impact of sand slugs against rigid targets, the results reported subsequently for impact of sand slugs against the buffer plates are also not sensitive to the choice of these inter-particle contact properties. Unless otherwise specified, the sand slug had a spatially uniform initial relative density $\bar{\rho}=0.2$.

We proceed now to detail the properties of the elastic block and the buffer plate. The elastic block was assumed was assumed to be made from an elastic solid with density 
$\rho_{m}=8000 \mathrm{kgm}^{-3}$, Young's modulus $E=210 \mathrm{GPa}$ and Poisson's ratio $v=0.3$ which is representative of steel. The thickness $c_{b}$ of the block and the height $H$ of the sand slug were varied in the parametric study reported in Section 3.3. The numerical calculations for the sand impact against the buffer plate were designed keeping in mind the regime maps presented in Section 2. In particular, in all the calculations reported here we kept $\psi=0.7, \bar{m}=1$ and $\bar{\rho}=0.2$ fixed and varied the nondimensional core height $\bar{H}$ and the core strength $\bar{\sigma}_{c}$ varied over a wide range. This was achieved by varying the parameters $H, c, \rho_{c}$ and $\sigma_{c}$ which will be specified on a case by case basis. We now proceed to detail the properties that remain fixed in all the calculations reported.

The buffer plate comprises a front face sheet of thickness $h=10 \mathrm{~mm}$ made from the same material as the elastic block described above, i.e. an elastic material with density $\rho_{m}=8000 \mathrm{kgm}^{-3}$, Young's modulus $E=210 \mathrm{GPa}$ and Poisson's ratio $v=0.3$, i.e. $m_{f}=8 \mathrm{kgm}^{-2}$. The core of density $\rho_{c}$ is modelled as a homogeneous compressible visco-plastic orthotropic foam-like material following Tilbrook et al. [27]. Assume the orthotropic axes $x_{i}$ of the core are aligned with the axes of the buffer plate as sketched in Fig. 3a, i.e $x_{1}$ and $x_{2}$ are aligned with the longitudinal and transverse directions, respectively. Introduce the stress and plastic strain matrices in the usual way as

$$
\boldsymbol{\sigma}=\left(\sigma_{1}, \sigma_{2}, \sigma_{3}, \sigma_{4}, \sigma_{5}, \sigma_{6}\right)^{T} \equiv\left(\sigma_{11}, \sigma_{22}, \sigma_{33}, \sigma_{13}, \sigma_{23}, \sigma_{12}\right)^{T},
$$

and

$$
\boldsymbol{\varepsilon}^{p}=\left(\varepsilon_{1}^{p}, \varepsilon_{2}^{p}, \varepsilon_{3}^{p}, \varepsilon_{4}^{p}, \varepsilon_{5}^{p}, \varepsilon_{6}^{p}\right)^{T} \equiv\left(\varepsilon_{11}^{p}, \varepsilon_{22}^{p}, \varepsilon_{33}^{p}, \varepsilon_{13}^{p}, \varepsilon_{23}^{p}, \varepsilon_{12}^{p}\right)^{T},
$$

respectively. Assume complete decoupling of material response between the orthogonal material directions and define the plastic strain rate $\dot{\varepsilon}_{i}^{p}$ via an overstress relation as

$$
\dot{\varepsilon}_{i}^{p}= \begin{cases}\left(\frac{\left|\sigma_{i}\right|-Y_{i}\left(\varepsilon_{i}^{p}\right)}{\eta}\right) & \text { if }\left|\sigma_{i}\right|>Y_{i}\left(\varepsilon_{i}^{p}\right) \\ 0 & \text { otherwise, }\end{cases}
$$

where the yield strength $Y_{i}\left(\varepsilon_{i}^{p}\right)$ is a function only of the plastic strain $\varepsilon_{i}^{p}$ and the material viscosity $\eta$ is taken to be a constant. The total strain rate $\dot{\varepsilon}_{i}$ is obtained by supplementing the above anisotropic plasticity model with isotropic elasticity such that

$$
\dot{\varepsilon}_{i}=L_{i j} \dot{\sigma}_{j}+\dot{\varepsilon}_{i}^{p} \operatorname{sign}\left(\sigma_{i}\right) \quad \text { (summation over } j \text { ). }
$$

In the case of isotropic elasticity, the compliance matrix $L_{i j}$ of the core material is specified in terms of the Young's modulus $E_{c}$ and Poisson's ratio $v_{c}$ as 


$$
\mathbf{L}=\left(\begin{array}{cccccc}
1 / E_{c} & -v_{c} / E_{c} & -v_{c} / E_{c} & 0 & 0 & 0 \\
& 1 / E_{c} & -v_{c} / E_{c} & 0 & 0 & 0 \\
& & 1 / E_{c} & 0 & 0 & 0 \\
& & 2\left(1+v_{c}\right) / E_{c} & 0 & 0 \\
& & & 2\left(1+v_{c}\right) / E_{c} & 0 \\
& & & & & 2\left(1+v_{c}\right) / E_{c}
\end{array}\right)
$$

We employ an isotropic elastic response for simplicity; this suffices as the core response of dictated by the plastic branch. The above elastic-plastic constitutive relation is expected to be adequate to model sandwich cores such as the squarehoneycomb core or the corrugated core; see for example Xue et al. [28].

In all the calculations reported here we assume an isotropic plastic response in the sense that the all the strengths $Y_{i}$ are assumed to be equal and strain hardening is neglected in all directions other than the transverse direction. The transverse strength $\sigma_{c}=Y_{2}$ is assumed to be independent of the plastic strain $\varepsilon_{2}^{p}$ up to a nominal densification strain $\varepsilon_{D}$ : beyond densification a linear hardening behaviour is assumed with a very large tangent modulus $E_{t}=0.1 E$. In all the calculations reported here the modulus and Poisson's ratio of the core were fixed at $E_{c}=20 \mathrm{GPa}$ and $v_{c}=0.25$, respectively and independent of the core density $\rho_{c}$. Further, consistent with the lumped parameter model calculations presented in Section 2 we assume that the densification strain is independent of the foam core density with $\varepsilon_{D}=0.8$. Finally, we note that the viscosity $\eta$ of the core was chosen such that the shock width (Radford et al., 2005)

$$
l=\frac{\eta \varepsilon_{D}}{\rho_{c} v_{o}}
$$

equals $c / 10$, with $v_{o}$ interpreted as the initial impact velocity of the sand particles. This prescription ensures that the shock width is always much less than the core depth yet is larger than the mesh size. Note that large gradients in stress and strain occur over the shock width and thus a mesh size smaller than $l$ is required to resolve these gradients accurately.

The buffer plates were discretised using quadrilaterals comprising four constant strain triangles with elements of size $h / 5$ and $c / 40$ in the face sheet and core, respectively while the element size employed in the elastic block was $c_{b} / 40$.

\subsection{Response of the reference elastic block}

One of the drawbacks of the lumped parameter model was that it did not include the effect of elasticity of the sand slug or the buffer plate and hence was unable to differentiate between the attached and unattached boundary conditions. In this section we discuss the response of a sand slug impact both the attached and unattached elastic block so as to illustrate the effects of elasticity in a relatively simple setting.

Dimensional analysis dictates that the spatial average pressure transmitted into the foundation, (for fixed sand particle contact properties) and is given by; 


$$
\bar{p}_{t} \equiv \frac{\varepsilon_{S} p_{t}}{\bar{\rho} \rho_{s} v_{o}^{2}}=f\left[\frac{c_{b}}{H}, \bar{\rho}, \frac{v_{o}}{\sqrt{E / \rho_{m}}}, \frac{\rho_{m}}{\rho_{s}}, \bar{t}, v\right],
$$

The spatial average pressure $p_{f}$ exerted by the sand slug over the impacted face of the elastic block is also a function of the same non-dimensional parameters and is thus given as

$$
\bar{p}_{f} \equiv \frac{\varepsilon_{S} p_{f}}{\bar{\rho} \rho_{s} v_{o}^{2}}=g\left[\frac{c_{b}}{H}, \bar{\rho}, \frac{v_{o}}{\sqrt{E / \rho_{m}}}, \frac{\rho_{m}}{\rho_{s}}, \bar{t}, v\right] .
$$

Here $\varepsilon_{S}$ is the densification strain of the sand slug and given by Eq. (2.16) to a very high degree of accuracy. While $p_{t}$ is calculated directly from the reaction force $R$ exerted by the plate on the foundation, $p_{t} \equiv R / W$ where $R$ is the reaction force exerted by the block per unit thickness in the $x_{3}$-direction, $p_{f}$ is calculated as follows. At any time $t$, there are $M$ sand particles in contact with the elastic block and $p_{f}$ is given as

$$
p_{f}(t)=\frac{\sum_{i=1}^{M} F_{2}^{i}}{W}
$$

where $F_{2}^{i}$ is the contact force (per unit depth in the $x_{3}$ direction) in the $x_{2}$ direction between the $i^{\text {th }}$ sand particle and elastic block.

The normalisations for the pressures in Eqs. (3.13) and (3.14) have been chosen so that $\bar{p}_{f}=\bar{p}_{t} \approx 1$ over the duration $0 \leq \bar{t} \varepsilon_{S} \leq 1$ for impact of the slug against a rigid plate (recall that Pingle et al. [15] established that the steady-state pressure exerted by the sand slug on a rigid stationary target is approximately $\bar{\rho} \rho_{s} v_{o}^{2} / \varepsilon_{S}$ and is exerted until the densification front reaches the distal end of the sand slug at time $\left.t=H \varepsilon_{S} / v_{o}\right)$. All results here are for a sand slug of height $H=10 \mathrm{~mm}$ impacting the elastic block with material properties detailed in Section 3.2.

First consider the unattached case. The predicted variation of the normalised pressures $\bar{p}_{f}$ and $\bar{p}_{t}$ versus the normalised time $\bar{t} \varepsilon_{S}$ are plotted in Figs. 8a and 8b for the choices $c_{b} / H=0.4$ and 50 , respectively and a sand impact velocity $v_{o}=400 \mathrm{~ms}^{-1}$ (i.e. $v_{o} / \sqrt{E / \rho_{m}}=0.08$ ). As will be clarified subsequently, the $c_{b} / H=0.4$ and 50 cases represent two limiting scenarios for the given sand slug loading. In the $c_{b} / H=0.4$ case, both $\bar{p}_{f}$ and $\bar{p}_{t}$ drop to zero at times $\bar{t} \varepsilon_{S}>1$. Over the period $0 \leq \bar{t} \varepsilon_{S} \leq 1, \bar{p}_{f}$ and $\bar{p}_{t}$ have an oscillatory response (the oscillations are larger in $\bar{p}_{f}$ ) but the mean values of both these pressure are approximately equal such that $\bar{p}_{f} \approx \bar{p}_{t} \approx 1^{3}$. These results indicate that $c_{b} / H=0.4$ block behaves

\footnotetext{
${ }^{3}$ These oscillations are a result of elastic wave reflections within the elastic block: when the elastic wave arrives at the sand/elastic block interface it partially reflects and causes a change
} 
approximately like a rigid body with multiple elastic wave reflections between the front and the rear end of the block resulting in approximate equilibrium conditions being attained, i.e. $\bar{p}_{f} \approx \bar{p}_{t}$. Thus, the $c_{b} / H=0.4$ case represents the limit where the loading time of the sand slug is much larger than the transit time for the elastic waves through the block. In this limit the elastic block may be approximated as a rigid body. Next consider the $c_{b} / H=50$ case. Again, $\bar{p}_{f} \approx 1$ over the $0 \leq \bar{t} \varepsilon_{S} \leq 1$ whereafter the pressure exerted by the sand slug reduces significantly. However, over this entire duration the transmitted pressure $\bar{p}_{t}=0$ as the elastic wave that initiates after the sand slug impacts the block at $t=0$ has not yet reached the rear end of the block at time $\bar{t} \varepsilon_{S}=1$. Infact, the elastic wave reaches the rear end of the block at time $\bar{t} \varepsilon_{S} \approx 5$, i.e. well after the sand slug has densified and bounced off the elastic block. This elastic wave then reflects from the block/foundation interface and thereby transmitting a pressure into the foundation equal to twice $\bar{p}_{f}$. The duration of this transmitted pressure is equal to the time taken for the sand slug to densify, i.e. the duration is approximately given by $H \varepsilon_{S} / v_{o}$. Hence over this period, $H \varepsilon_{S} / v_{o}$, the momentum transmitted into the foundation is twice the momentum transferred by the sand slug into the elastic block. The reflected compressive wave then travels towards the front end of the elastic block and reflects as a tensile wave from the free surface. Upon reaching the rear of the block, the tensile wave results in loss of contact between the block and the foundation with the block "bouncing-off" the foundation (recall no tensile stresses can be transmitted across that interface in this unattached case). The final momentum transmitted into the supports is thus twice the momentum of the incoming sand slug, with the additional transferred momentum accounted for by the bounce-off of the elastic block. Thus, the $c_{b} / H=50$ case represents the limit where the loading time of the sand slug is much less than the transit time for the elastic waves through the block. In this limit the loading may be approximated as impulsive and results in a significant rebound of the elastic block.

Now consider the attached case. The temporal predictions of $\bar{p}_{f}$ and $\bar{p}_{t}$ are included in Figs. 9a and $9 \mathrm{~b}$ for the $c_{b} / H=0.4$ and 50, respectively. Consistent with the fact that the $c_{b} / H=0.4$ behaves similar to a rigid block, the predictions of the pressures $\bar{p}_{f}$ and $\bar{p}_{t}$ are nearly identical over the time period of the sand slug loading, i.e. $0 \leq \bar{t} \varepsilon_{S} \leq 1$. Subsequently, $\bar{p}_{f}$ drops to zero as the sand slug bounces off the elastic block. However, elastic waves continue to reflect within the elastic block resulting in alternating compressive and tensile forces being transmitted into the support resulting in the oscillatory nature of the $\bar{p}_{t}$ response for $\bar{t} \varepsilon_{S}>1$ as seen in Fig. 9a. Of course these oscillatory $\bar{p}_{t}$ do not on average result in any additional momentum being transmitted into the support for $\bar{t} \varepsilon_{S}>1$. The differences between the attached and unattached cases become more apparent for the $c_{b} / H=50$. The responses of the

in velocity of that interface and hence a change in the contact pressure between the sand particle and the elastic block. 
attached and unattached cases are similar up to $\bar{t} \varepsilon_{S} \approx 15$. At this point the tensile stress wave impinges on the interface between the block and the support. In the unattached case this resulted in loss of contact between the block and the support with the block bouncing off the support. However, in the attached case the interface can sustain tension which results in a rectangular negative $\bar{p}_{t}$ pulse with a magnitude and duration equal to the earlier compressive pulse. This pulse thus cancels out the momentum that was transmitted by that earlier compressive pulse resulting in the net momentum transmitted into the support temporarily reducing to zero just after the end of this tensile pulse. However, with no dispersion of the wave within the elastic block, the tensile and compressive pulses continue to get alternatively transmitted into the foundation.

The predictions of transmitted areal momentum

$$
I_{t}(t)=\int_{0}^{t} p_{t} d t
$$

normalised by the incoming momentum $I_{o}\left(\bar{I}_{t} \equiv I_{t} / I_{o}\right)$ of the sand slug are included in Figs. 10a and $10 \mathrm{~b}$ for the $c_{b} / H=0.4$ and 50 cases respectively for both the attached and unattached cases. Consistent with the transmitted pressure histories plotted in Figs. 8a and 9a for the $c_{b} / H=0.4$ case, $I_{t}$ increases monotonically and plateaus-out at $I_{t} / I_{o} \approx 1$ with the extra $5 \%$ transmitted momentum due to rebound of the sand particles as discussed by Pingle et al. [15]. Further, the results are nearly identical for the attached and unattached cases expect for the small oscillations in the attached case about the steady-state value $I_{t} / I_{o}$ due to the fact that $p_{t}$ oscillates between positive and negative values due to internal reflections of elastic waves as seen in Fig. 9a. By contrast, the build-up of momentum $I_{t}$ in the attached and unattached $c_{b} / H=50$ blocks are markedly different. The $I_{t} / I_{o}$ curves are identical until the tensile elastic reflection discussed above reaches the rear end of the block at $\bar{t} \varepsilon_{S} \approx 15$. Thereafter the unattached block loses contact with the foundation and the momentum transferred into the foundation remains constant at $I_{t} / I_{o} \approx 2$. However, in the attached case the transmitted pressure is now tensile which results in a reduction in $I_{t}$ to zero. The transmitted momentum $I_{t} / I_{o}$ then oscillates periodically between 0 and 2 in-sync with the compressive and tensile elastic wave reflections within the elastic block.

We define $I_{t}^{\text {avg }}$ as the time-averaged transmitted momentum (where we average over 10 oscillations in the $I_{t}$ versus time curves). Predictions of $\bar{I}_{t}^{\text {avg }} \equiv I_{t}^{\text {avg }} / I_{o}$ are plotted in Fig. 11 as a function of the quantity

$$
\bar{\tau} \equiv \frac{\varepsilon_{S} H}{c_{b} v_{o}} \sqrt{\frac{E}{\rho_{m}}} .
$$

Here $\bar{\tau}$ the ratio of the time taken for the sand slug to densify (i.e. the time over which the sand slug exerts a pressure) to the time taken for an elastic wave to travel from the front to the rear end of the elastic block. The discussion above suggests that this ratio of time-scales is the key parameter that governs the momentum transferred 
into the foundation, especially in the unattached case. Results are included in Fig. 11 for two values of the normalised sand impact velocity $v_{o} / \sqrt{E / \rho_{m}}=0.04$ and 0.08 corresponding to $v_{o}=400 \mathrm{~ms}^{-1}$ and $200 \mathrm{~ms}^{-1}$, respectively for both the attached and unattached cases.

First consider the attached case. The transmitted momentum $I_{t}^{\text {avg }} / I_{o} \approx 1$ over the entire parameter range considered here, i.e. $I_{t}^{a v g} / I_{o}$ is independent of $\bar{\tau}$ and $v_{o} / \sqrt{E / \rho_{m}}$. Next consider the unattached case. Here $I_{t}^{a v g} / I_{o}$ displays a strong dependence on $\bar{\tau}$ and the two limits discussed above are clearly seen: $\bar{\tau}>10$ corresponds to the rigid block limit with $I_{t}^{a v g} / I_{o} \approx 1$ while the $\bar{\tau}<1$ limit is impulsive limit with $I_{t}^{a v g} / I_{o} \approx 2$. At intermediate values of $\bar{\tau}, I_{t}^{a v g} / I_{o}$ increases with decreasing $\bar{\tau}$. Again, there is only a mild dependence on $v_{o} / \sqrt{E / \rho_{m}}$. Detailed numerical calculations were performed to confirm that $I_{t}^{a v g} / I_{o}$ is only dependent on the boundary conditions (viz. attached or unattached) and the ratio of time-scales $\bar{\tau}$ but reasonably independent of all the other non-dimensional groups in Eqs. (3.13) and (3.14). We note in passing here that while $I_{t}^{a v g} / I_{o}$ differ for the attached and unattached cases in the $\bar{\tau}<1$ limit the maximum transmitted transient momentum are equal in both these cases with $I_{t}^{\max } / I_{o} \approx 2$.

\subsection{Selected numerical results for the buffer plate in the four regimes}

We proceed to illustrate the responses of both the attached and unattached buffer plates by first presenting numerical calculations for the four geometries labelled G-I through G-IV in Fig. 5a, i.e. $\bar{\rho}=0.2, \psi=0.7$ and $\bar{m}=1$ the parameters are held fixed. In these calculations the sand slugs have a height $H=\psi m_{f} /\left(\bar{\rho} \rho_{s}\right)=10 \mathrm{~mm}$ while the values of the parameters $c, \rho_{c}$ and $\sigma_{c}$ for geometries G-I through G-IV are chosen to achieve the appropriate values of $\bar{\sigma}_{c}$ and $\bar{H}$. All calculations were performed for a sand impact velocity $v_{o}=400 \mathrm{~ms}^{-1}$.

We characterise the responses of buffer plates in terms of the following five quantities:

(i) the core compression defined as $\varepsilon_{c} \equiv \Delta c / c$, where $\Delta c$ is the reduction in the overall thickness of the core;

(ii) the pressure $p_{t}$ at the interface between the foam core and the foundation;

(iii) the pressure $p_{f}$ exerted by the sand slug on the front face of the sandwich plate; and

(iv) the momentum $I_{t}$ transferred into the foundation.

The core compression predictions for both the attached and unattached cases are nearly indistinguishable and hence only predictions of the temporal evolution of $\varepsilon_{c}$ for the unattached buffer plates are included in Fig. 6a along with the predictions of the lumped parameter model. Note that the FE calculations predict no permanent core compression for geometry G-IV (only a small transient elastic compression of the 
core). Hence results for geometry G-IV are not included in Fig. 6a. We now discuss in detail in responses of the unattached buffer plates and then subsequently illustrate the key differences that occur when the buffer plates are attached to the foundation.

Geometry $G$-I in regime I: The temporal variation of the normalised pressures $\bar{p}_{t} \equiv p_{t} \varepsilon_{s} /\left(\bar{\rho} \rho_{s} v_{o}^{2}\right)$ and $\bar{p}_{f} \equiv p_{f} \varepsilon_{s} /\left(\bar{\rho} \rho_{s} v_{o}^{2}\right)$ are plotted in Fig. 12a for the unattached case, while snapshots showing the deformation of the buffer plate and sand slug at four selected values of time $\bar{t}$ are included in Fig. 12b. For times $\bar{t}<0.3$, both $p_{f}$ and $p_{t}$ are approximately constant. During this time a densification wave traverses through the core and hence the buffer plate only transmits a stress $p_{t} \approx \sigma_{c} \ll p_{f}$ into the foundation. Further, the pressure $p_{f}$ exerted by the sand on the buffer plate is also approximately constant with $p_{f} \approx\left(\bar{\rho} \rho_{s} v_{o}^{2}\right) / \varepsilon_{s}$ as the front face acquires a rather small velocity. At $\bar{t} \approx 0.3$, the densification front reaches the rear resulting in the socalled "slap" event where the front face slaps into the foundation through the now completely densified core. This results in a sudden increase in $p_{t}$ to levels significantly above $\left(\bar{\rho} \rho_{s} v_{o}^{2}\right) / \varepsilon_{s}$. An elastic compressive wave now traverses from the rear to the front face. The wave then imparts a velocity in the positive $x_{2}$-direction to the front face that results in the observed sudden increase in the interfacial pressure $p_{f}$. This compressive wave reflects from the interface between the front face sheet and the sand slug as a tensile wave which upon reaching the rear face of the buffer plate results in the plate bouncing off the foundation. However, there is continued loading of the buffer plate by the sand slug which results in multiple impacts and rebounds of the densified buffer plate against the foundation. This continues until $\bar{t} \approx 1$ when the sand slug also densifies. Subsequently, the sand slug rebounds off the buffer plate and both the buffer plate and sand slug now acquire a steady-state velocity so that contact between the sand slug and the plate and between the plate and the foundation is not established again.

The corresponding temporal evolution of the normalised core compression $\varepsilon_{c} / \varepsilon_{D}$ and transmitted momentum $I_{t} / I_{o}$ are included in Figs. 6a and 13a, respectively. We clearly observe that there is negligible momentum transfer prior to core densification at $\bar{t} \approx 0.3$. Subsequently, $I_{t}$ builds-up in three distinct spurts corresponding to the spikes in $p_{t}$ seen in Fig. 12a, i.e. momentum transfer in geometry G-I is governed by the "slap" event and the subsequent impacts of the buffer plate against the foundation. However, the maximum momentum transferred to the foundation $I_{t}^{\max } / I_{o} \approx 1$, i.e. equal to the incoming momentum of the sand slug.

Geometry GII in regime II: The temporal variation of the normalised pressures $\bar{p}_{t}$ and $\bar{p}_{f}$ are plotted in Fig. 14a while snapshots showing the deformation of the buffer plate and sand slug at four selected values of time $\bar{t}$ are included in Fig. 14b. Just after impact, the sand slug exerts a pressure $p_{f} \approx 1$ on the buffer plate. However, $\bar{p}_{f}$ now reduces with increasing $\bar{t}$ as the front face acquires a velocity $v_{f}$ comparable to 
$v_{o}$. At $\bar{t} \approx 1$, the sand slug fully densifies as the densification front within the sand slug reaches the top of the slug and there is a sudden drop in the interfacial pressure $\bar{p}_{f}$. The densification front within the sand slug reflects from the top end as a tensile front resulting in sand spalling-off the top end of the slug; see Fig. 14b. Meanwhile, the densification wave initiated in the core continues to transverse towards the rear face resulting in continued core compression. During this time the buffer plate transmits a pressure $p_{t} \approx \sigma_{c}$ to the foundation (recall that $\sigma_{c} \ll \bar{\rho} \rho_{S} v_{0}^{2}$ that thus in Fig. $14 \mathrm{~b}$ it seems as if $\left.p_{t} \approx 0\right)$. At $\bar{t} \approx 3$, the densification front within the core reaches the rear end resulting in a "slap" event and a sudden increase in both $p_{t}$ and $p_{f}$ similar to that discussed for geometry G-I. Shortly thereafter, the buffer plate rebounds off the foundation and a steady-state (with no contact between the buffer plate and the foundation or the sand slug and the buffer plate) is attained. Unlike geometry G-I, multiple impacts between the buffer plate and the foundation do not occur as the sand slug had densified and hence stopped loading the plate prior to core densification. This results in the majority of the momentum transfer to the foundation occurring during the single slap event that occurs at the instant of full densification; see Figs. 14a and 13a. Note that the total momentum transfer is significantly higher compared to geometry G-I (in geometry G-II, $I_{t}^{\max } / I_{o} \approx 1.5$ compared to $I_{t}^{\max } / I_{o} \approx 1.1$ for geometry G-I).

Geometry G-III in regime III: The temporal variation of the normalised pressures $\bar{p}_{t}$ and $\bar{p}_{f}$ are plotted in Fig. 15a while snapshots showing the deformation of the buffer plate and sand slug at four selected values of time $\bar{t}$ are included in Fig. 15b. Similar to geometry GII, the pressure $\bar{p}_{f}$ reduces with time as the velocity $v_{f}$ increases and then suddenly drops to approximately zero at $\bar{t} \approx 1$ when the densification front within the sand slug reaches the top end of the slug. However, the stronger core in geometry G-III compared to geometry G-II, results in the deceleration of the front face and hence reloading of the buffer plate by the sand slug as seen in Fig. 15a. This reloading however results in significantly lower pressures compared to the loading prior to the densification of the sand slug. The pressure $p_{t}$ exerted by the buffer plate on the foundation is equal to the strength $\sigma_{c}$ of the foam core over the entire duration of the impact event- the densification front in the core arrests before it reaches the foundation and hence there is no dramatic increase in $p_{t}$ as in cases G-I and G-II.

The deformation of the buffer plate without full densification (the maximum value of $\mathcal{E}_{c}$ is approximately 0.3 as seen from Fig. 3a) results in a "soft catch" mechanism whereby the buffer plate slowly brings the sand slug to rest. This reduces the spalling of the sand so that $I_{t}^{\max } / I_{o} \approx 1$ as seen in Fig. 13a, i.e. there is effectively a perfectly plastic collision between the sand slug and the buffer plate. We note in passing that momentum conservation dictates that $I_{t}^{\max } / I_{o} \geq 1$ and thus this geometry G-III gives the minimum momentum transfer to the supports for the given sand slug loading. 
Geometry G-IV in regime $I V$ : This geometry has a sufficiently strong core that no plastic deformation initiates within the core under these impact conditions. The problem thus reduces to the impact of the sand slug against an elastic plate resting on a rigid foundation. Thus, $\bar{p}_{f} \approx 1$ until the sand slug fully densifies at $\bar{t} \approx 1$ whereafter the pressure drops to zero (Fig. 16a). The transmitted pressure $p_{t}$ displays large oscillations with time due to elastic stress waves running between the front face and the foundation (Fig. 16a). These elastic stress waves also get partially transmitted into the sand slug and cause the low amplitude oscillations seen in the $p_{f}$ time traces. Snapshots showing the deformation of the sand slug included in Fig. 16b confirm that the situation is equivalent to the impact of the sand slug against a rigid plate.

For geometry G-IV, the maximum transmitted momentum into the foundation $I_{t}^{\max } / I_{o} \approx 1.15$ (Fig. 13a), and is equal to that transmitted into a rigid foundation (i.e. the elasticity does not affect the total momentum transfer to any significant extent as G-IV is in the $\bar{\tau} \approx 1$ regime). As discussed above, the transmitted momentum is greater than $I_{o}$ due to the spalling of the sand which results in the sand slug bouncing off the buffer plate. There is no permanent core compression for geometry G-IV with the calculations predicting only elastic core compression strains less than about $1 \%$. We thus do not include the core compression predictions in Fig. 3.

The main difference between the attached and unattached cases is that the attached buffers plates do not loose contact with the foundation and can transmit a tensile force. The front face pressures $p_{f}$ for the four attached geometries G-I through G-IV are nearly identical to the unattached cases plotted in Figs. 12a, 14a, 15a and 16a. However, the transmitted pressures differ, with the pressures in the attached case becoming tensile over the time intervals where $p_{t}=0$ due to loss of contact between the buffer plate and the foundation in the unattached case. The corresponding predictions of the temporal evolution of the transmitted momentum $I_{t} / I_{o}$ are included in Fig. 13b. Unlike the unattached case (Fig. 13a), $I_{t}$ can decrease with increasing time due to the transmission of tensile forces into the foundation. In fact, the steady-state situation always corresponds to an oscillating $I_{t}$ versus time response due to continued elastic wave reflections within the buffer plate after the sand slug has lost contact with the plate.

\subsection{Predictions of transmitted momentum}

We now present detailed predictions of the transmitted momentum for the buffer plate geometries considered in the map in Fig. 5a, viz. $\bar{\rho}=0.2, \psi=0.7$ and $\bar{m}=1$. Predictions of $I_{t}^{a v g} / I_{o}$ as a function of the normalised core strength $\bar{\sigma}_{c}$ are included in Figs. 17a and 17b for the unattached and attached cases, respectively. (Recall that $I_{t}^{a v g}$ is defined as the average momentum over 10 periodic oscillations of the $I_{t}$ versus time curve.) The limit of the sand slug impacting the rigid stationary foundation is also included in Fig. 17 with $I_{t}^{a v g} / I_{o} \approx 1.1$ due to the rebound of the sand slug.

The salient observations from Figs. $17 \mathrm{a}$ and $17 \mathrm{~b}$ include: 
(i) The transmitted momentum $I_{t}^{\text {avg }} / I_{o} \geq 1$ for both the attached and unattached cases.

(ii) Typically $I_{t}^{\text {avg }} / I_{o}$ is at its minimum at intermediate values of the normalised core strength $\bar{\sigma}_{c}$. In this regime there is incomplete densification of the sandwich core which results in the "soft catch" mechanism. This minimises bounce-back of the sand slug and minimizes the momentum transfer.

(iii) In the unattached case, for both large and small values of $\bar{\sigma}_{c}$ there is significant bounce-back of both the buffer plate and sand slug that results in $I_{t}^{a v g} / I_{o}$ rising to values close to the elastic impact limit of $I_{t}^{\text {avg }} / I_{o}=2$.

(iv) In the attached case, for large values of $\bar{\sigma}_{c}, I_{t}^{a v g} / I_{o}$ equals the rigid foundation limit as the buffer plate with the strong core does not deform. On the other hand, for small values of $\bar{\sigma}_{c}$, a slap event occurs which results in the sand slug bouncing back with a higher velocity as discussed above but the buffer plate of course remaining attached to the foundation. Thus, $I_{t}^{a v g} / I_{o}$ in this case is lower compared to the unattached case where there is no transmission of tensile forces into the foundation. However, it is worth mentioning here that $I_{t}^{\max } / I_{o}$ for both the attached and unattached cases are nearly identical as this transient maximum momentum transfer occurs just prior to the tensile reflection reaching the rear of the buffer plate.

(v) Typically $I_{t}^{\text {avg }} / I_{o}$ is higher for lower values of $\bar{H}$ as the sand slug densifies before the end of core compression giving rise to a more impulsive load on the buffer plate. This impulsive type load results in more bounce-back and hence the larger values of $I_{t}^{a v g} / I_{o}$.

\subsection{Comparison between the lumped parameter model and FE predictions}

Comparison between the lumped parameter model and predictions of the coupled discrete/FE calculations are made for the following metrics: (i) regime boundaries and the (ii) core compression $\varepsilon_{c}$. We do not explicitly show comparisons of the transmitted momentum as the lumped parameter model predicts $I_{t}^{\max } / I_{o}=I_{t}^{a v g} / I_{o}=1$ in all cases: the above numerical results clearly show that this is in general a poor prediction with the transmitted momentum depending on numerous factors including attachment conditions, face and core elasticity, core height etc. This clearly demonstrates that the lumped parameter model is inadequate in predicting the transmitted momentum.

The maps in Figs. 5a and 5b show the regime boundaries as predicted by the lumped parameter model. We performed an extensive numerical study and conducted approximately 100 coupled FE/discrete calculations over each of these maps. These calculations were distributed throughout the map but we chose about 40 geometries near the regime boundaries marked in Fig. 5. These spot calculations near the regime boundaries marked in Fig. 5 demonstrated that the regime boundaries predicted by the lumped parameter model are nearly indistinguishable from those predicted by the coupled discrete/FE analysis. Further the coupled numerical calculations predicted that the regime boundaries are insensitive to the attachment conditions. Thus, for the 
sake of clarity we only include regime boundaries as predicted by the lumped parameter model in all maps shown in this study.

Predictions of the temporal evolution of $\varepsilon_{c}$ for geometries G-I through G-III are included in Fig. 6a using both the lumped parameter model and the coupled discrete/FE simulations for both the attached and unattached cases. Excellent agreement is observed between the lumped parameter and FE predictions. Moreover, consistent with the fact that the regime boundaries are insensitive to the attachment conditions, the temporal evolution of the core compression is also insensitive to the attachment conditions (and hence the lines for the attached and unattached discrete/continuum predictions are indistinguishable in Fig. 6a). Further evidence of the fidelity of the lumped parameter model in accurately capturing the maximum core compression is seen in Fig. $6 \mathrm{~b}$ where we plot the normalised core strain $\varepsilon_{c}^{\max } / \varepsilon_{D}$ as a function of the normalised core strength $\bar{\sigma}_{c}$ for selected values of $\bar{H}$ keeping $\bar{\rho}=0.2, \psi=0.7$ and $\bar{m}=1$ fixed, i.e. equal to those for the regime map in Fig. 5a. Again we observe that the lumped parameter model predictions are in excellent agreement with the coupled discrete/FE predictions.

\section{Concluding remarks}

The response of back-supported compound buffer plates consisting of a foam core and solid face sheet subjected to an impact by a column of high velocity sand particles (sand slug) is investigated both via a semi-analytical lumped parameter model and coupled discrete/continuum simulations. The lumped model is used to construct deformation regime maps with axes of the normalised core strength and the ratio of the heights of the sand slug and the crushable core. Four regimes of behaviour are identified based on the level of core compression and the sequence of events, i.e. whether core compression or sand slug densification occurs first.

Selected discrete/continuum simulations are also reported in the four regimes identified by the lumped parameter model. The discrete/continuum simulations confirm that the lumped parameter model accurately predicts (i) the core compression of the buffer plate and (ii) the regimes of behaviour. However, the lumped parameter model neglects elasticity in the sand slug, the face sheets and core of the buffer plate. This results in the lumped parameter model not accurately predicting the momentum transfer into the foundation. Moreover, the lumped parameter model cannot differentiate between a panel that is attached or just resting on a foundation whereas the simulations indicate significant differences between these two boundary conditions.

The numerical calculations predict that the momentum transfer from the sand slug to the foundation is minimised for intermediate values of the core strength when the socalled "soft-catch" mechanism is at play. In this case, the buffer plate minimises bounce-back of the sand slug which in turn reduces the momentum transfer by up to $10 \%$. For high values of the core strength, the response of the buffer plate resembles a rigid plate with nearly no impulse mitigation while at low values of core strength, a slap event occurs when the face sheet impinges against the foundation due to full densification of the foam core. This slap event results in a significant enhancement of the momentum transfer to the foundation. The results reported here demonstrate that 
appropriately designed buffer plates have the potential as impulse mitigators in landmine loading situations.

\section{Acknowledgements}

This research was supported by the Office of Naval Research (ONR NICOP grant number N00014-09-1-0573) as part of a Multidisciplinary University Research Initiative on "Cellular Materials Concepts for Force Protection", Prime Award No. N00014-07-1-0764 is gratefully acknowledged. The program manager was Dr David Shifler.

\section{References}

[1] Fleck, N.A. and Deshpande, V.S. (2004). The resistance of clamped beams to shock loading, Journal of Applied Mechanics, 71, 386-401.

[2] Xue, Z. and Hutchinson, J.W. (2004). A comparative study of blast-resistant metal sandwich plates, International Journal of Impact Engineering 30(10): 12831305.

[3] Wadley, H.N.G., Dharmasena, K.P., Chen, Y., Dudt P., Knight, D., Charette, R., and Kiddy, K. (2008) Compressive Response of Multilayered Pyramidal Lattice During Underwater Shock Loading, International Journal of Impact Engineering, 35, 1102-1114.

[4] Wei, Z., Deshpande, V.S., Evans, A.G., Dharmasena, K.P., Queheillalt, D.T., Wadley, H.N.G., Murty, Y., Elzey, R.K., Dudt, P., Chen, Y., Knight, D. and Kiddy, K (2008). The resistance of metallic plates to localized impulse. Journal of Mechanics and Physics of Solids, 56, 2074-2091.

[5] Kambouchev, N., Noels, L., and Radovitzky, R. (2007). Fluid-structure interaction effects in the loading of free-standing plates by uniform shocks, Journal of Applied Mechanics, 74, 1042-1045.

[6] Taylor, G.I. (1963). The pressure and impulse of submarine explosion waves on plates. The Scientific Papers of Sir Geoffrey Ingram Taylor, Vol. III: Aerodynamics and the Mechanics of Projectiles and Explosions, G. Batchelor, ed., Cambridge University Press, Cambridge, UK, 287-303.

[7] Deshpande, V.S., and Fleck, N.A. (2005). One-dimensional response of sandwich plates to underwater shock loading, Journal of the Mechanics and Physics of Solids, 53, 2347-2383.

[8] Deshpande, V.S., Heaver, A., and Fleck, N.A. (2006). An underwater shock simulator, Proceedings of the Royal Society A, 462, 1021-1041.

[9] Espinosa, H.D., and Lee, S., and Moldovan, N. (2006). A novel fluid structure interaction experiment to investigate deformation of structural elements subjected to impulsive loading, Experimental Mechanics, 46, 805-824. 
[10] Hutchinson, J.W. (2009). Energy and Momentum Transfer in Air Shocks, Journal of Applied Mechanics, 76, 051307-1-7.

[11] Dharmasena, K.P., Wadley, H.N.G., Xue, Z., and Hutchinson, J.W., (2008). Mechanical response of metallic honeycomb sandwich panel structures to high intensity dynamic loading, International Journal of Impact Engineering, 35, 11021114

[12] Dharmasena, K.P., Wadley, H.N.G., Williams, K., Xue, Z., and Hutchinson, J.W., (2011). Response of Metallic Pyramidal Lattice Core Sandwich Panels to High Intensity Impulsive Loading in Air, International Journal of Impact Engineering, 38, pp. $275-289$

[13] Wang, Z., Hao, H. and Lu, Y. (2004). A three-phase soil model for simulating stress wave propagation due to blast loading, International Journal for Numerical and Analytical Methods in Geomechanics, 28, 33-56.

[14] Borvik, T., Olovsson, L., Hanssen, A.G., Dharmasena, K., Hansson, H. and Wadley, H.N.G. (2011). A discrete particle approach to simulate the combined effect of blast and sand impact loading of steel plates, Journal of the Mechanics and Physics of Solids, 59, 940-958.

[15] Pingle, S.M., Fleck, N.A., Wadley, H.N.G. and Deshpande, V.S. (2012). Discrete element calculations of the impact of a sand column against rigid structures, International Journal of Impact Engineering, 45, 74-89.

[16] Liu, T., Fleck, N.A., Deshpande, V.S. and Wadley, H.N.G. (2012). The impact of sand slugs against beams and plates: coupled discrete/continuum calculations. Submitted.

[17] Wadley, H.N.G., Børvik, T., Olovsson, L., Wetzel, J.J., Dharmasena, K.P., Hopperstad, O.S., Deshpande, V.S. and Hutchinson, J.W. (2012). Deformation and fracture of impulsively loaded sandwich panels, To appear in the Journal of the Mechanics and Physics of Solids.

[18] Deshpande, V.S. and Fleck, N.A. (2000). Isotropic constitutive models for metallic foams, Journal of the Mechanics and Physics of Solids, 48, 1253-1283.

[19] Ashby, M.F., Evans, A.G., Fleck, N.A., Gibson, L.J., Hutchinson, J.W. and Wadley, H.N.G. (2000). Metal foams: a design guide, Butterworth-Heinemann, Boston MA.

[20] Wadley, H.N.G., Dharmasena, K., Chen, Y.C., Dudt, P., Knight, D., Charette, R., and Kiddy, K. (2008). Compressive response of multilayered pyramidal lattices during underwater shock loading. International Journal of Impact Engineering, 35, 1102-1114.

[21] Reid, S.R., and Peng, C. (1997). Dynamic uniaxial crushing of wood. International Journal of Impact Engineering, 19, 531-570. 
[22] Plimpton, S. (1995). Fast parallel algorithms for short-range molecular dynamics. Journal of Computational Physics 117, 1-19.

[23] Cundall, P.A. and Strack, O.D.L. (1979). A discrete numerical model for granular assemblies. Geotechnique, 29, 47-65.

[24] Campbell, C.S., and Brennen, C.E. (1985). Chute flows of granular material: some computer simulations. Journal of Applied Mechanics, ASME, 52, 172-178.

[25] Campbell, C.S. (2002). Granular shear flows at the elastic limit. Journal of Fluid Mechanics, 465, 261-291.

[26] Silbert L.E., Ertaş D., Grest G.S., Halsey, T.C., Levine, D., Plimpton, S.J. (2001). Granular flow down an inclined plane: Bagnold scaling and rheology. Physics review $E, 64,051302$.

[27] Tilbrook, M.T., Deshpande, V.S. and Fleck, N.A. (2006). The impulsive response of buffer plates: Analytical and numerical investigation of the regimes of behaviour, Journal of the Mechanics and Physics of Solids, 54, 2242-2280.

[28] Xue, Z., Vaziri, A. and Hutchinson, J.W. (2005). Non-uniform hardening constitutive model for compressible orthotropic materials with application to sandwich plate cores, Computer Modelling and Simulation in Engineering, 10, 79-96.

\section{Figure Captions}

Fig. 1: Sketch of a prototypical problem of a clamped sandwich structure loaded by a shallow mine explosion.

Fig. 2: (a) High speed photographs showing the soil ejecta resulting from the detonation of a landmine buried under water saturated soil. (b) Sketch of the experimental set-up used to investigate the response of back-supported buffer plates subjected to a landmine explosion.

Fig. 3: (a) Sketch of the boundary value problem analysed for the impact of a sand column against the buffer plate or elastic block. The constraint, which prevents the sand slug from laterally expanding, is not shown for the sake of clarity. Sketches showing the leading dimensions of (b) buffer plate and (c) elastic block are also included.

Fig. 4: The idealised uniaxial compressive stress versus strain curves of (a) the foam core of the buffer plate and (b) the sand slug. (c) Sketch of the one-dimensional shock propagation in the foam core and the sand slug after impact.

Fig. 5: Deformation regime maps predicted by the lumped parameter model for the parameter choice (a) $\psi=0.7$ and (b) $\psi=10$ with $\bar{m}=1$ and $\bar{\rho}=0.2$. Contours of the normalised maximum core compression $\varepsilon_{c}^{\max } / \varepsilon_{D}$ are included in regime III. In (a) the four reference cases analysed are marked while in (b) the regime boundaries for $\psi=0.7$ are also included. 
Fig. 6: Predictions of (a) time evolution of normalised core compression $\varepsilon_{c} / \varepsilon_{D}$ for the reference cases G-I, G-II and G-III and (b) the maximum normalised core compression $\varepsilon_{c}^{\max } / \varepsilon_{D}$ as a function of the normalised core strength $\bar{\sigma}_{c}$ for selected values of the normalised core height $\bar{H}(\bar{m}=1, \bar{\rho}=0.2$ and $\psi=0.7)$. Predictions using both the lumped parameter and the discrete/continuum models are presented for both the attached and unattached cases.

Fig. 7: Sketch illustrating the contact law between two particles in the discrete calculations.

Fig. 8: Discrete/continuum simulation predictions of the time evolution of the normalised pressures $\bar{p}_{f}$ and $\bar{p}_{t}$ for the unattached elastic block with (a) $c_{b} / H=0.4$ and (b) $c_{b} / H=50$ for the normalised sand slug velocity $v_{o} / \sqrt{E / \rho_{m}}=0.08$.

Fig. 9: Discrete/continuum simulation predictions of the time evolution of the normalised pressures $\bar{p}_{f}$ and $\bar{p}_{t}$ for the attached elastic block with (a) $c_{b} / H=0.4$ and (b) $c_{b} / H=50$ for the normalised sand slug velocity $v_{o} / \sqrt{E / \rho_{m}}=0.08$.

Fig. 10: Discrete/continuum simulation predictions of the time evolution of the normalised transmitted momentum $\bar{I}_{t}$ for the attached and unattached elastic block with (a) $c_{b} / H=0.4$ and (b) $c_{b} / H=50$ for the normalised sand slug velocity $v_{o} / \sqrt{E / \rho_{m}}=0.08$.

Fig. 11: Discrete/continuum simulation predictions of the normalised average transmitted momentum $I_{t}^{a v g} / I_{o}$ as a function of the time-scale ratio $\bar{\tau}$ for the attached and unattached elastic block. Results are presented for two values of the non-dimensional sand slug velocity $v_{o} / \sqrt{E / \rho_{m}}$.

Fig. 12: Discrete/continuum simulation predictions for the unattached buffer plate G-I. (a) Time evolution of the normalised front and transmitted pressures $\bar{p}_{f}$ and $\bar{p}_{t}$, respectively. (b) Snapshots showing the deformation of the buffer plate and sand slug at selected normalised times $\bar{t}$. The $*$ symbol indicates loss of contact between the buffer plate and foundation while the buffer plate face sheets are shown with hatch marks.

Fig. 13: Time evolution of the normalised transmitted momentum $\bar{I}_{t}$ for the (a) unattached and (b) attached buffer plate geometries G-I through G-IV.

Fig. 14: Discrete/continuum simulation predictions for the unattached buffer plate G-II. (a) Time evolution of the normalised front and transmitted pressures $\bar{p}_{f}$ and $\bar{p}_{t}$, respectively. (b) Snapshots showing the deformation of the buffer plate and sand slug 
at selected normalised times $\bar{t}$. The $*$ symbol indicates loss of contact between the buffer plate and foundation while the buffer plate face sheets are shown with hatch marks.

Fig. 15: Discrete/continuum simulation predictions for the unattached buffer plate G-III. (a) Time evolution of the normalised front and transmitted pressures $\bar{p}_{f}$ and $\bar{p}_{t}$, respectively. (b) Snapshots showing the deformation of the buffer plate and sand slug at selected normalised times $\bar{t}$. The ${ }^{*}$ symbol indicates loss of contact between the buffer plate and foundation while the buffer plate face sheets are shown with hatch marks.

Fig. 16: Discrete/continuum simulation predictions for the unattached buffer plate G-IV. (a) Time evolution of the normalised front and transmitted pressures $\bar{p}_{f}$ and $\bar{p}_{t}$, respectively. (b) Snapshots showing the deformation of the buffer plate and sand slug at selected normalised times $\bar{t}$. The ${ }^{*}$ symbol indicates loss of contact between the buffer plate and foundation while the buffer plate face sheets are shown with hatch marks.

Fig. 17: Discrete/continuum simulation predictions of the normalised average transmitted momentum $I_{t}^{\text {avg }} / I_{0}$ as function of the normalised core strength $\bar{\sigma}_{c}$ for selected values of $\bar{H}$ with $\bar{m}=1, \bar{\rho}=0.2$ and $\psi=0.7$, i.e. case considered in Fig. 5a. The unattached and attached cases are shown in (a) and (b) 


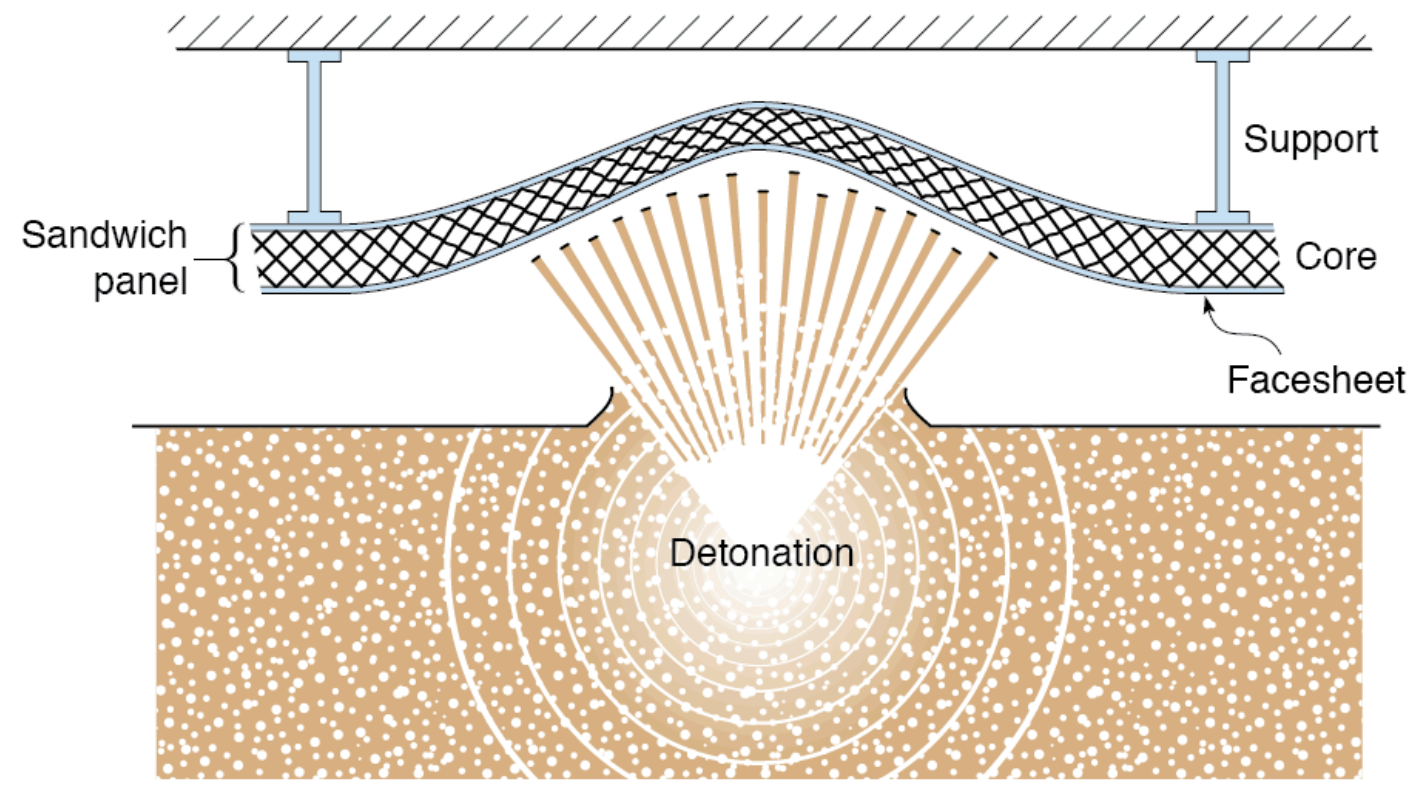

Fig. 1: Sketch of a prototypical problem of a clamped sandwich structure loaded by a shallow mine explosion. 
(a)

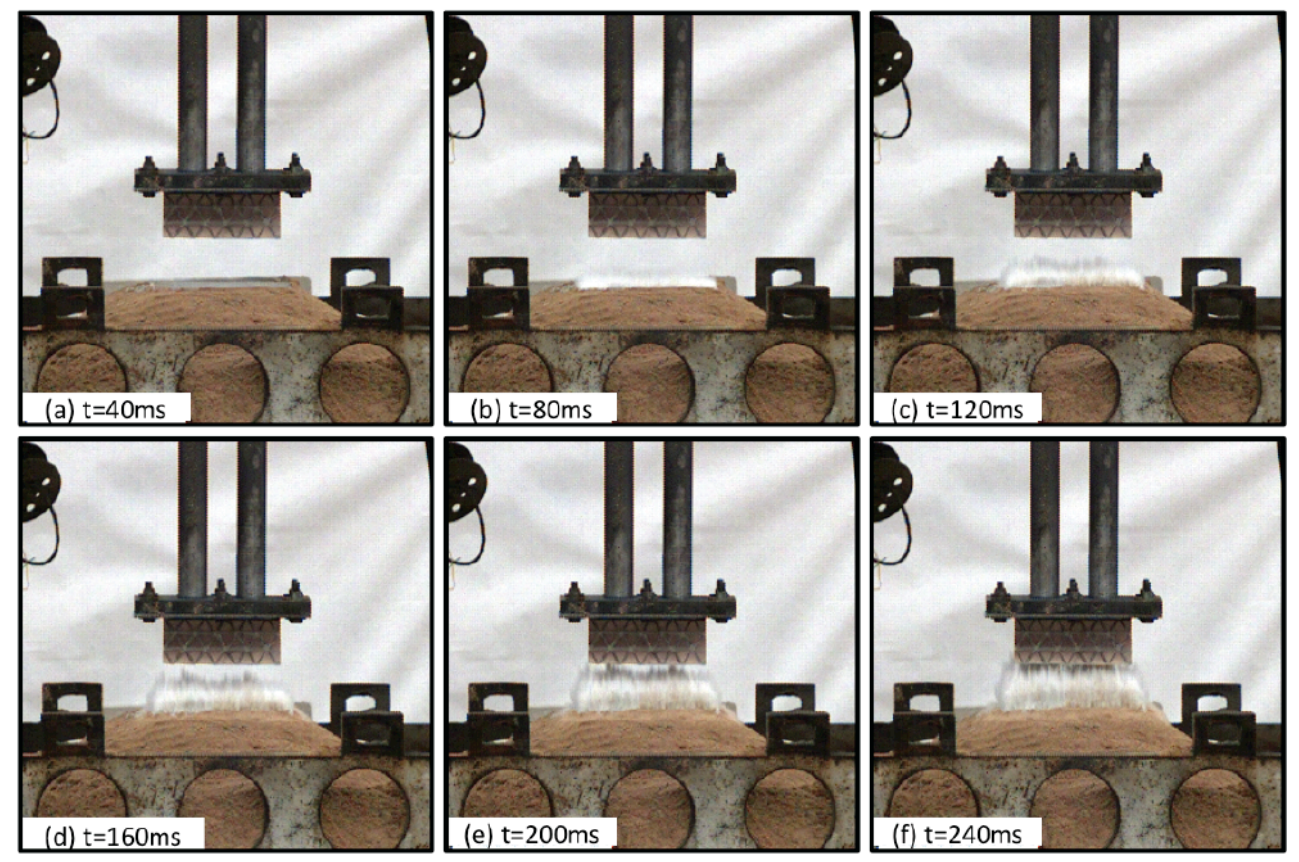

(b)

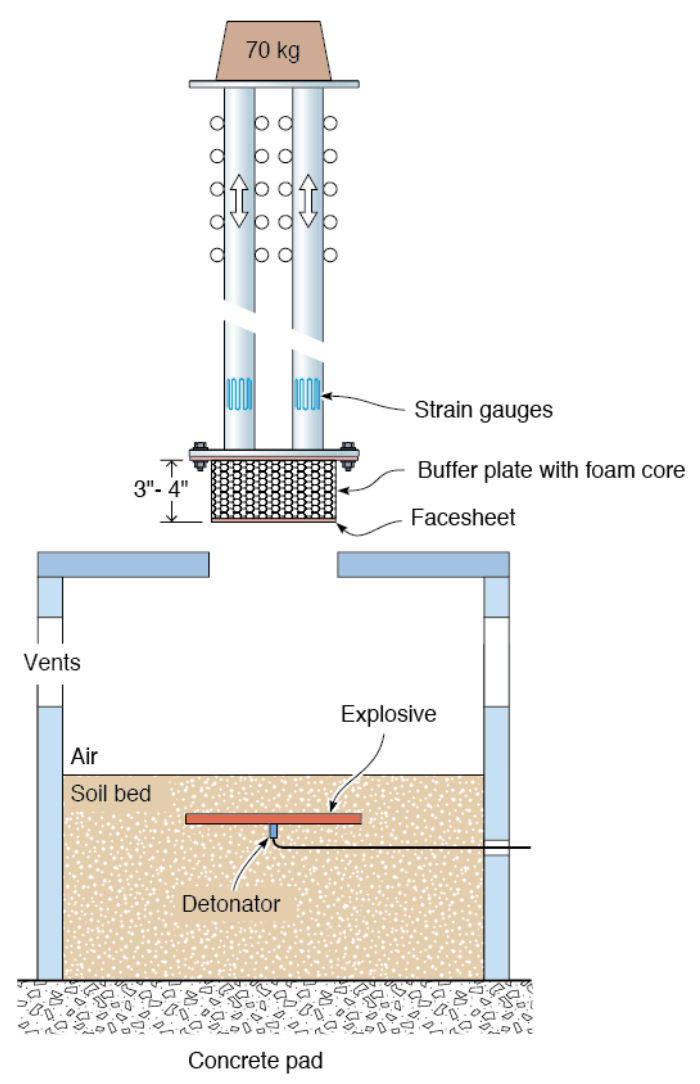

Fig. 2: (a) High speed photographs showing the soil ejecta resulting from the detonation of a landmine buried under water saturated soil. (b) Sketch of the experimental set-up used to investigate the response of back-supported buffer plates subjected to a landmine explosion. 


\section{(a)}

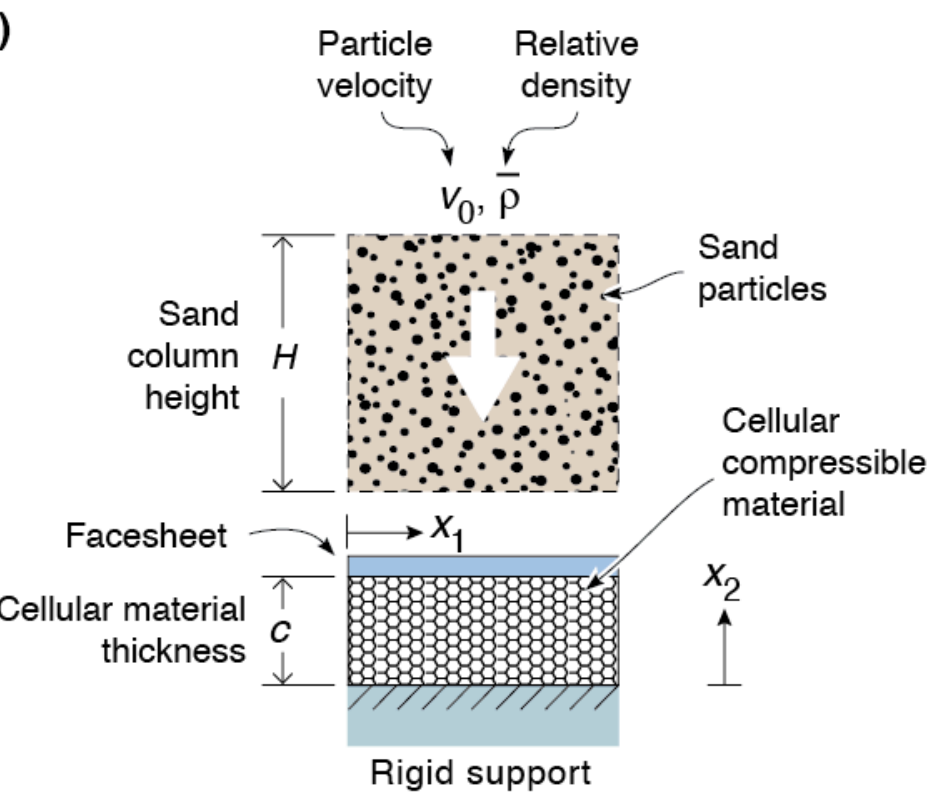

(b)

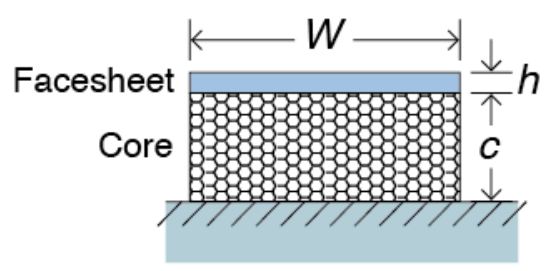

(c)

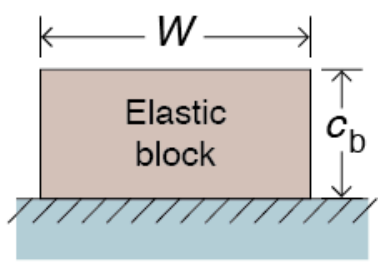

Fig. 3: (a) Sketch of the boundary value problem analysed for the impact of a sand column against the buffer plate or elastic block. The constraint, which prevents the sand slug from laterally expanding, is not shown for the sake of clarity. Sketches showing the leading dimensions of (b) buffer plate and (c) elastic block are also included. 
(a)

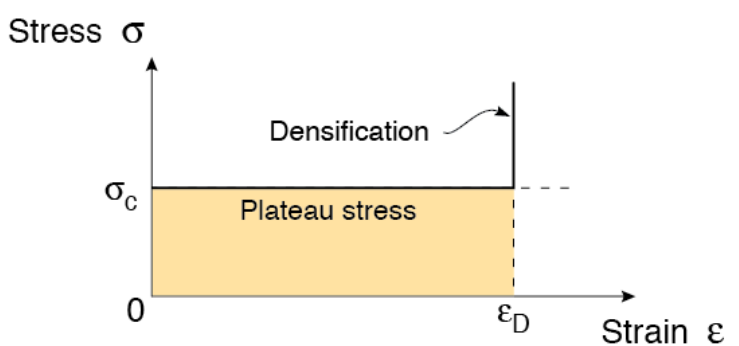

(b)

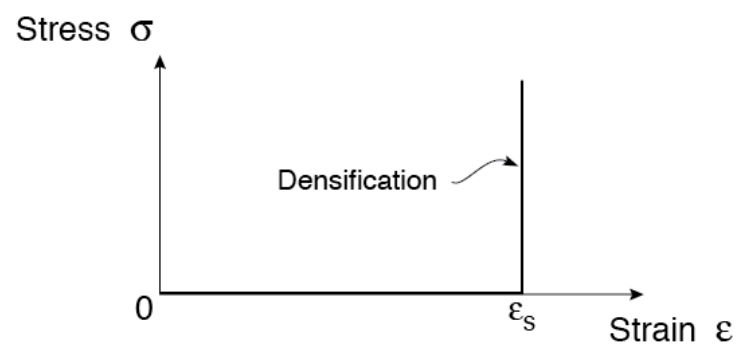

(c)

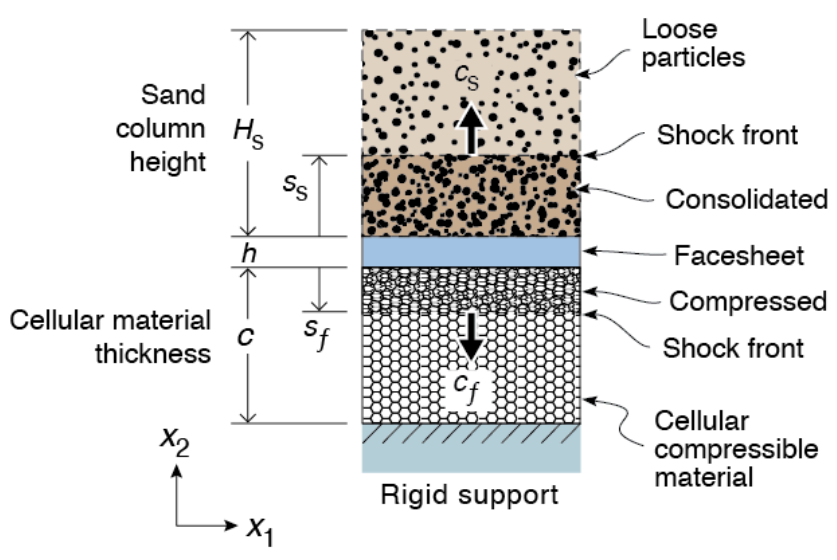

Fig. 4: The idealised uniaxial compressive stress versus strain curves of (a) the foam core of the buffer plate and (b) the sand slug. (c) Sketch of the one-dimensional shock propagation in the foam core and the sand slug after impact. 
(a)

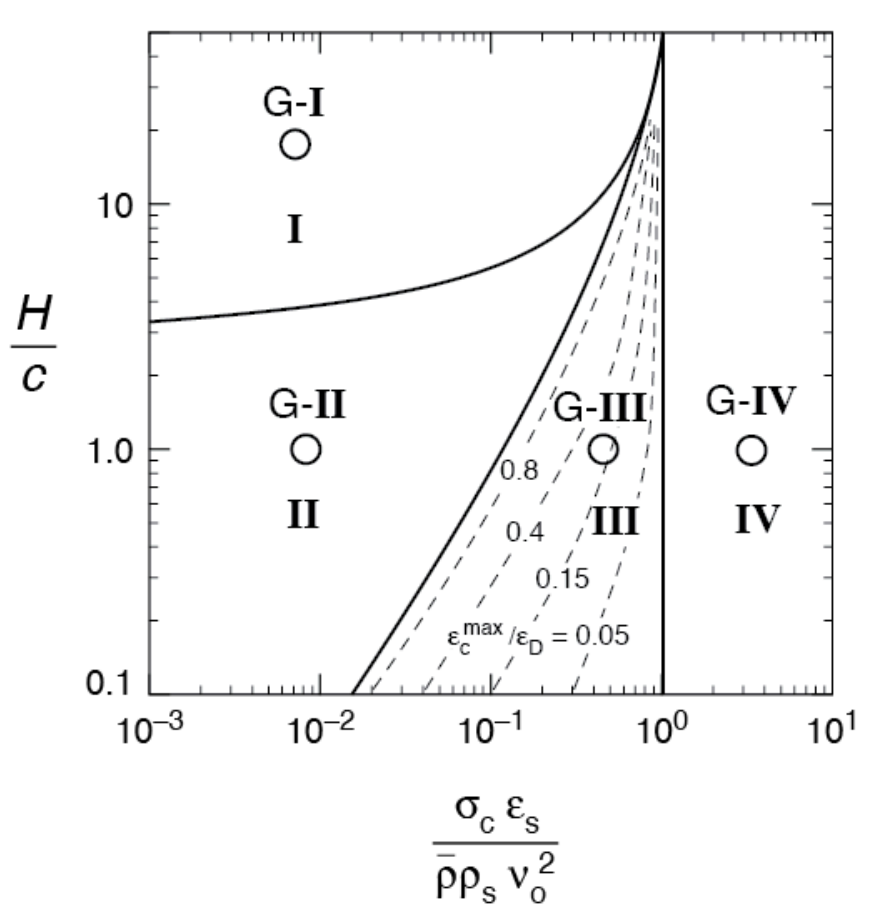

(b)

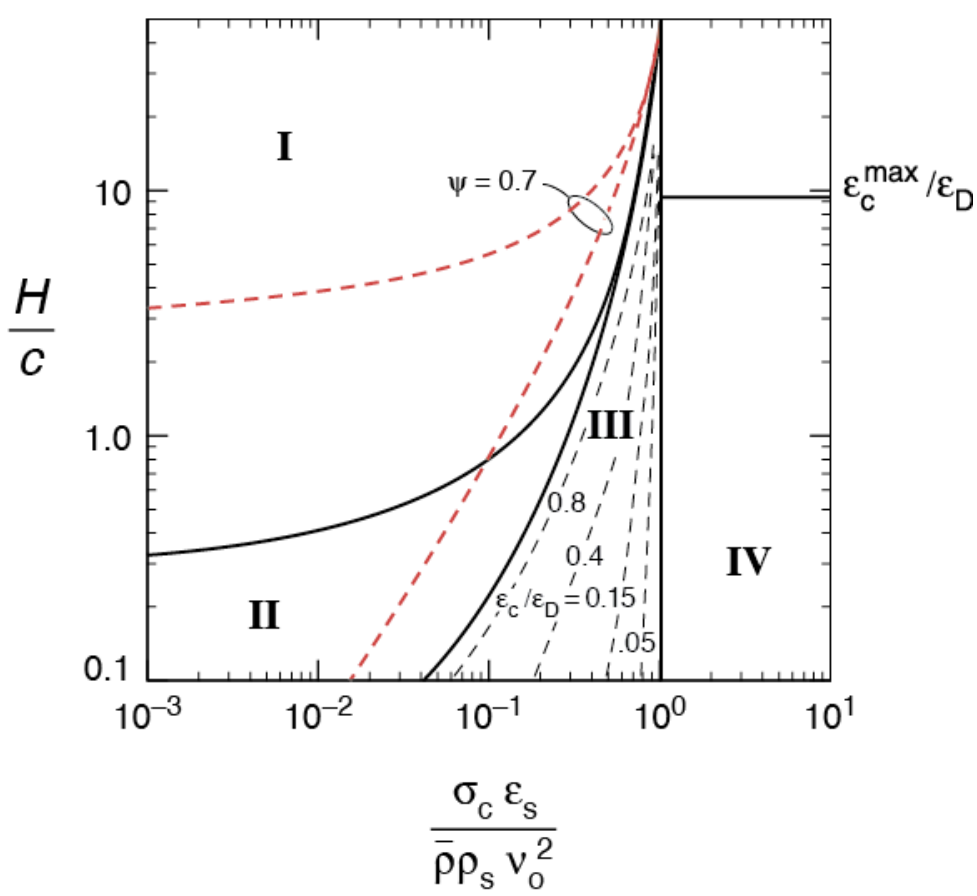

Fig. 5: Deformation regime maps predicted by the lumped parameter model for the parameter choice (a) $\psi=0.7$ and (b) $\psi=10$ with $\bar{m}=1$ and $\bar{\rho}=0.2$. Contours of the normalised maximum core compression $\varepsilon_{c}^{\max } / \varepsilon_{D}$ are included in regime III. In (a) the four reference cases analysed are marked while in (b) the regime boundaries for $\psi=0.7$ are also included. 
(a)

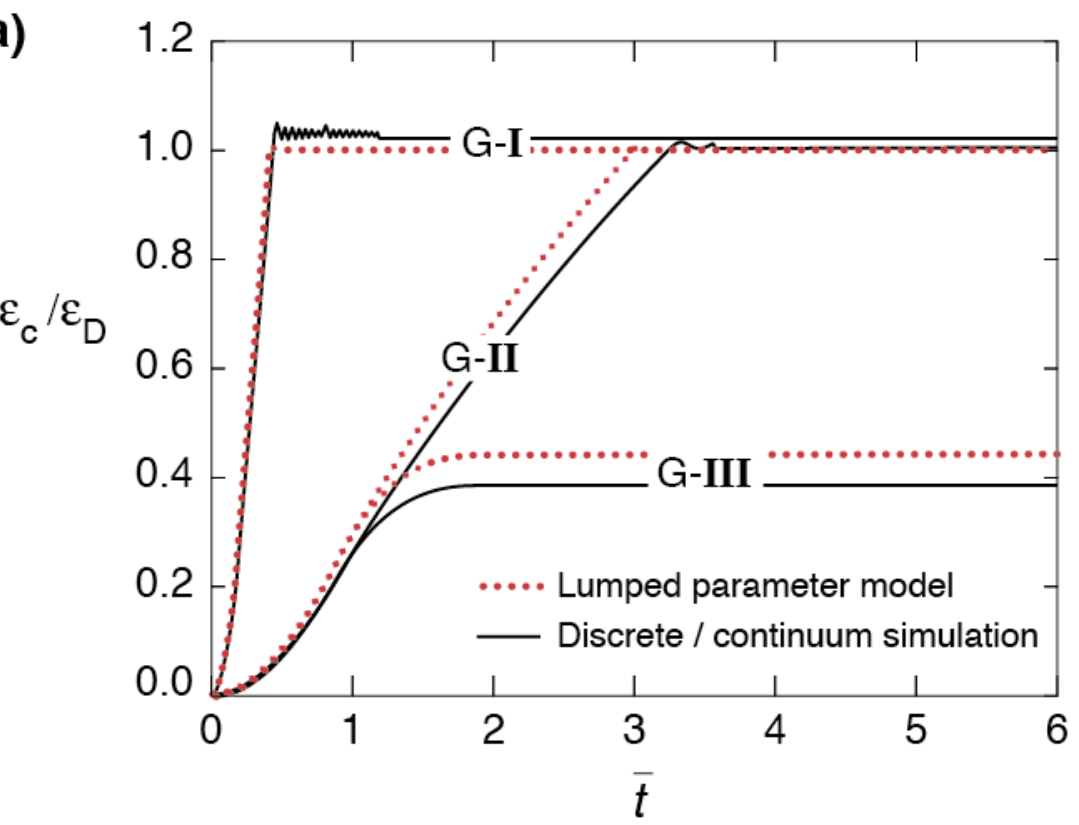

(b)

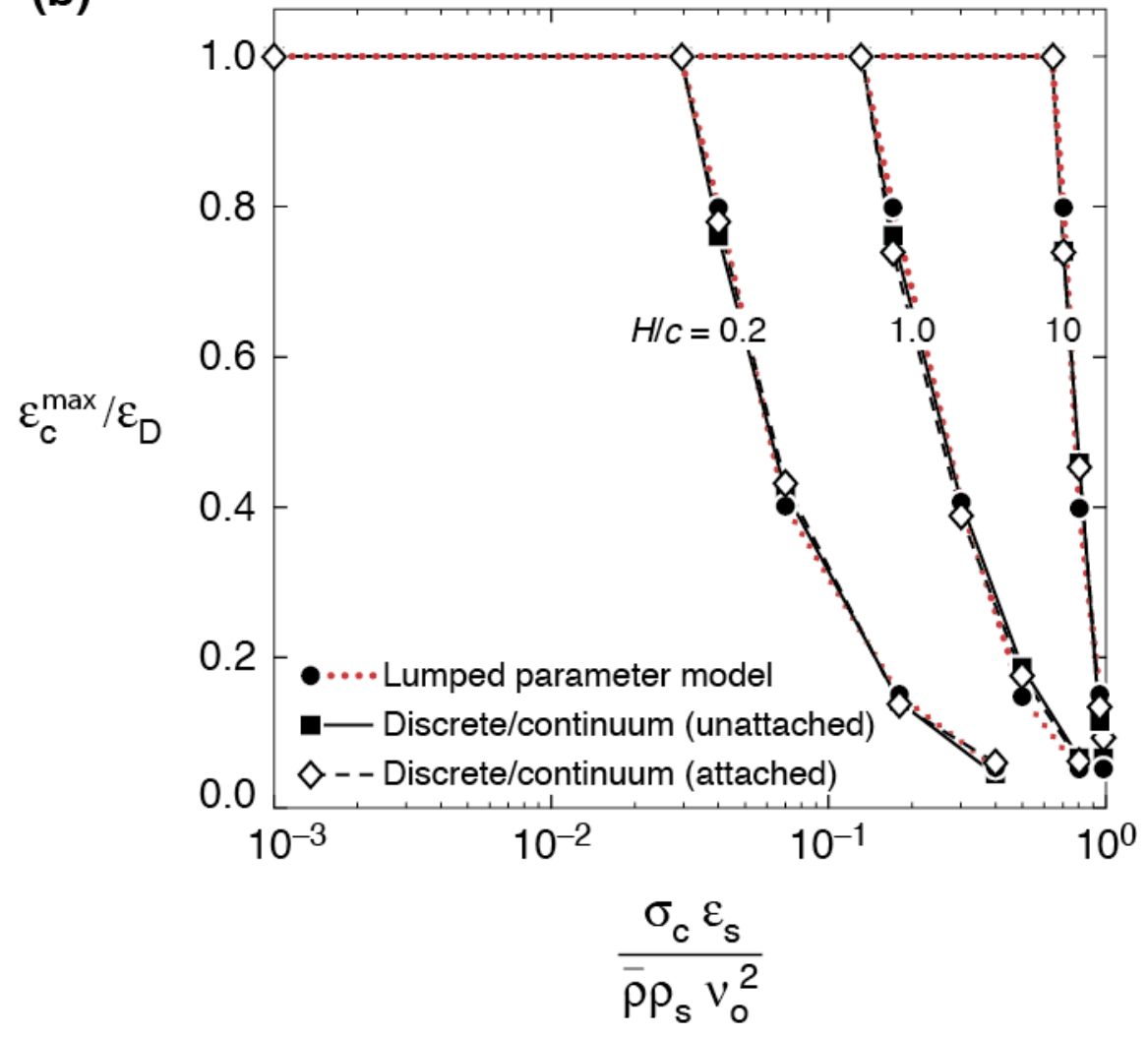

Fig. 6: Predictions of (a) time evolution of normalised core compression $\varepsilon_{c} / \varepsilon_{D}$ for the reference cases G-I, G-II and G-III and (b) the maximum normalised core compression $\varepsilon_{c}^{\max } / \varepsilon_{D}$ as a function of the normalised core strength $\bar{\sigma}_{c}$ for selected values of the normalised core height $\bar{H}(\bar{m}=1, \bar{\rho}=0.2$ and $\psi=0.7)$. Predictions using both the lumped parameter and the discrete/continuum models are presented for both the attached and unattached cases. 


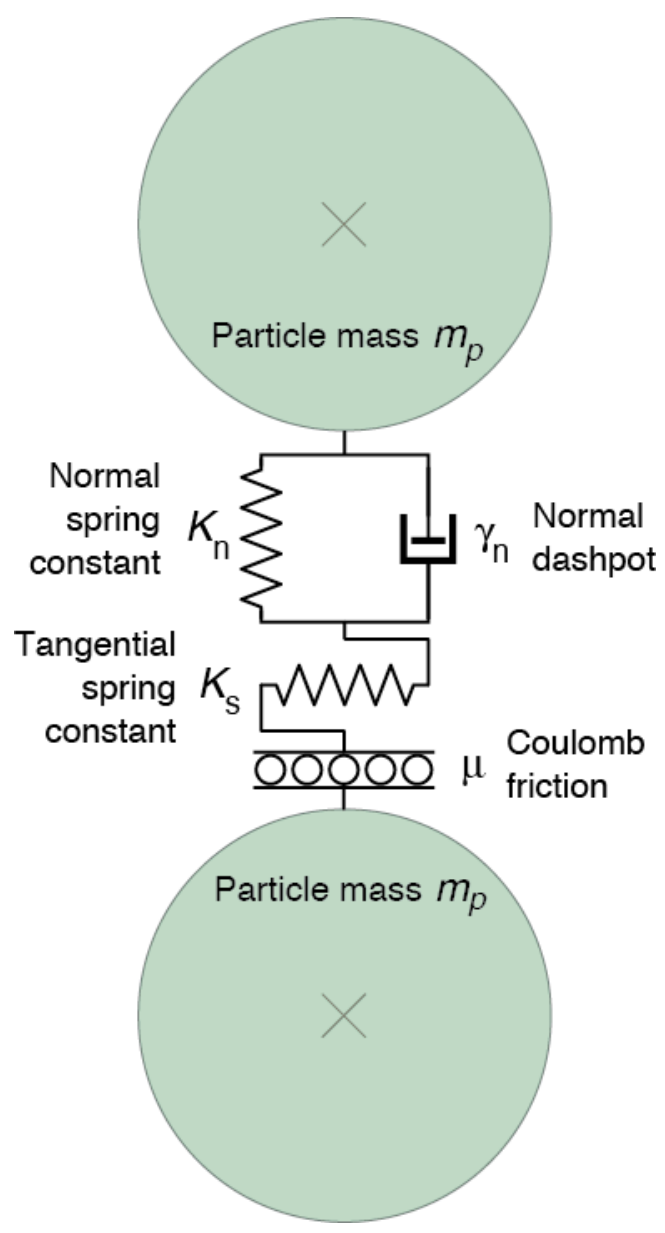

Fig. 7: Sketch illustrating the contact law between two particles in the discrete calculations. 
(a)

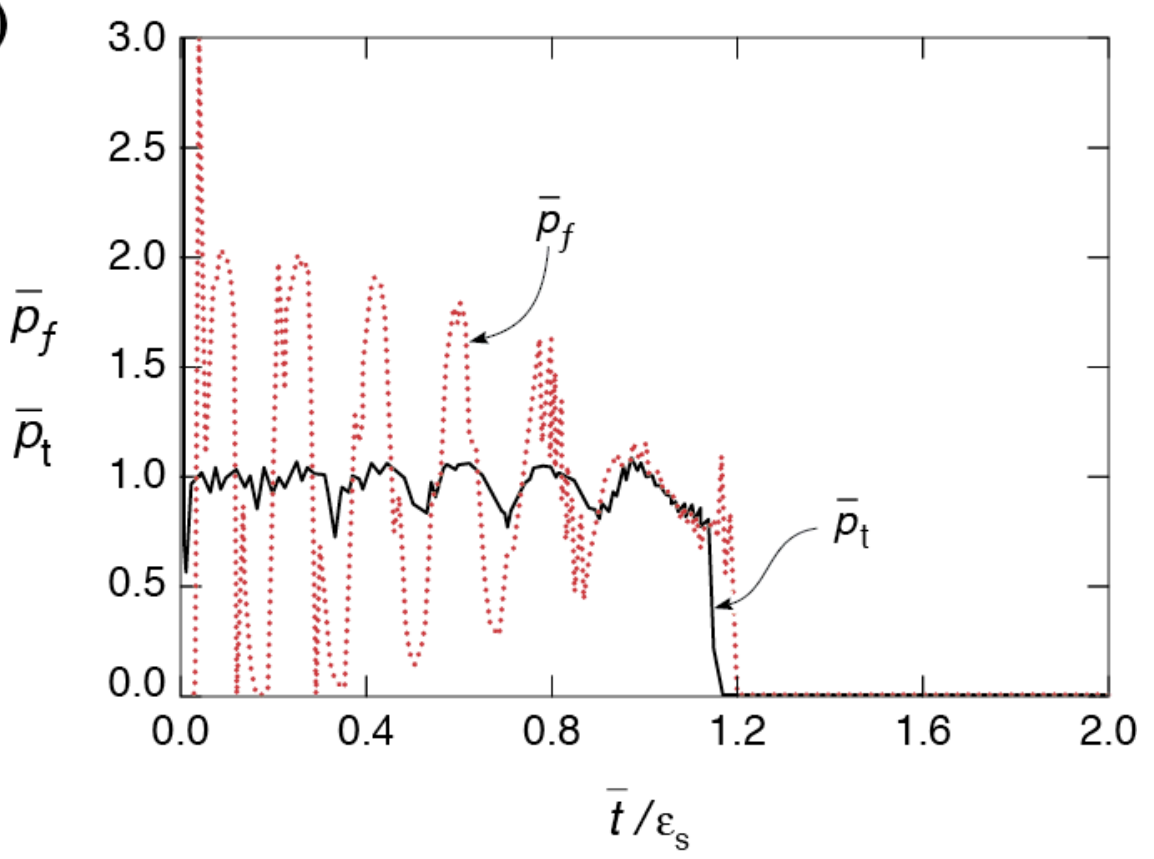

(b)

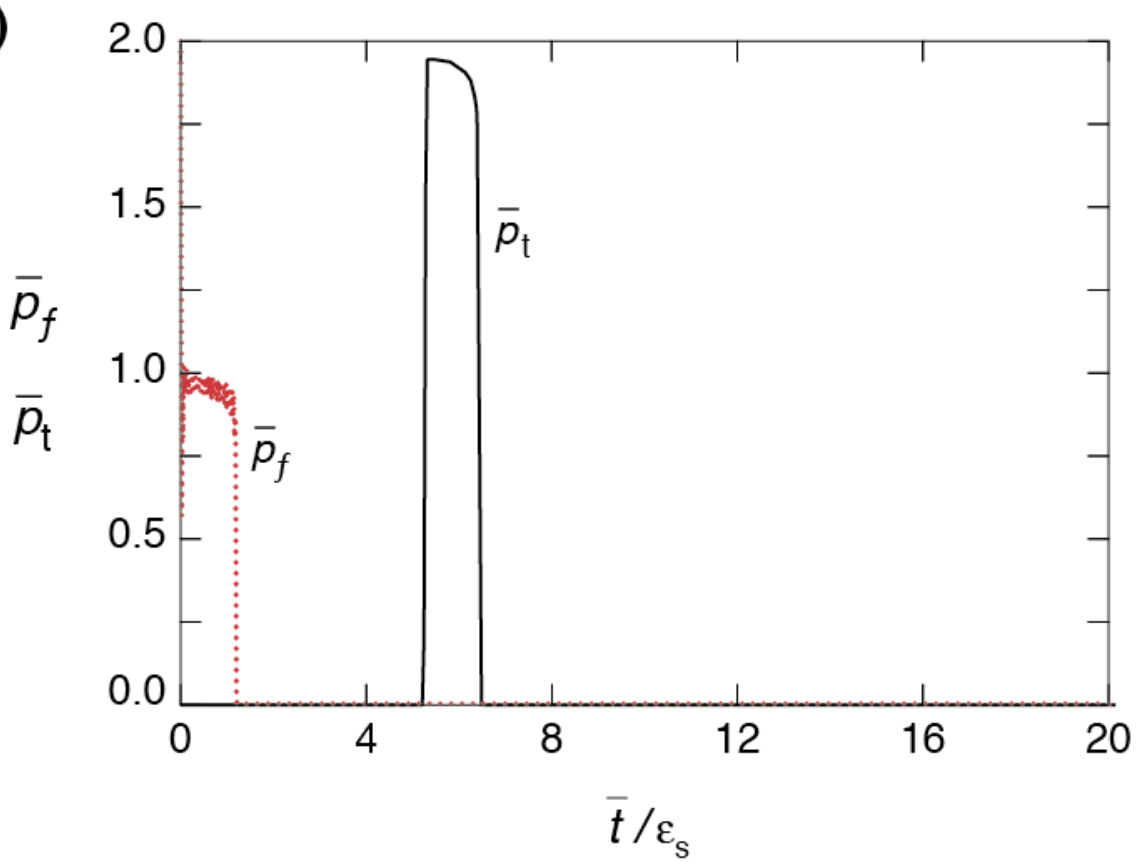

Fig. 8: Discrete/continuum simulation predictions of the time evolution of the normalised pressures $\bar{p}_{f}$ and $\bar{p}_{t}$ for the unattached elastic block with $c_{b} / H=0.4$ and (b) $c_{b} / H=50$ for the normalised sand slug velocity $v_{o} / \sqrt{E / \rho_{m}}=0.08$. 
(a)

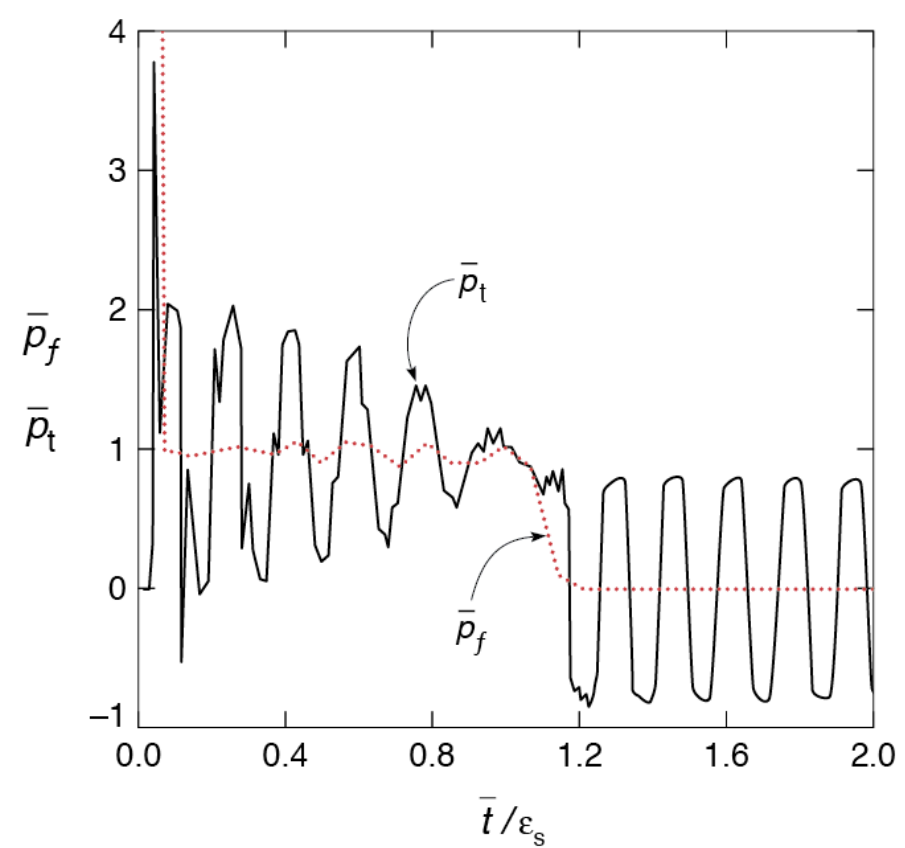

(b)

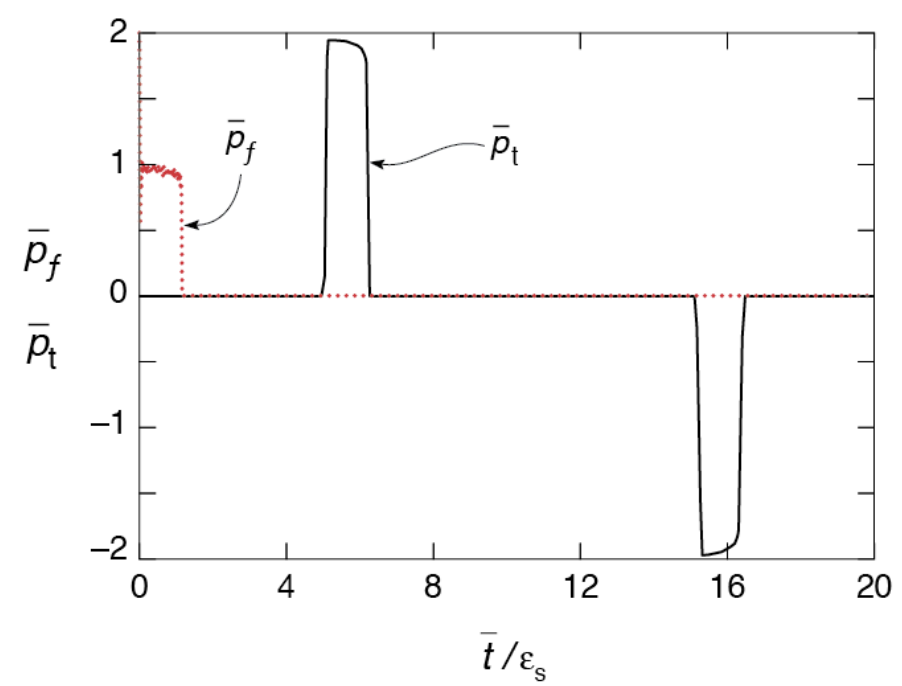

Fig. 9: Discrete/continuum simulation predictions of the time evolution of the normalised pressures $\bar{p}_{f}$ and $\bar{p}_{t}$ for the attached elastic block with (a) $c_{b} / H=0.4$ and (b) $c_{b} / H=50$ for the normalised sand slug velocity $v_{o} / \sqrt{E / \rho_{m}}=0.08$. 
(a)

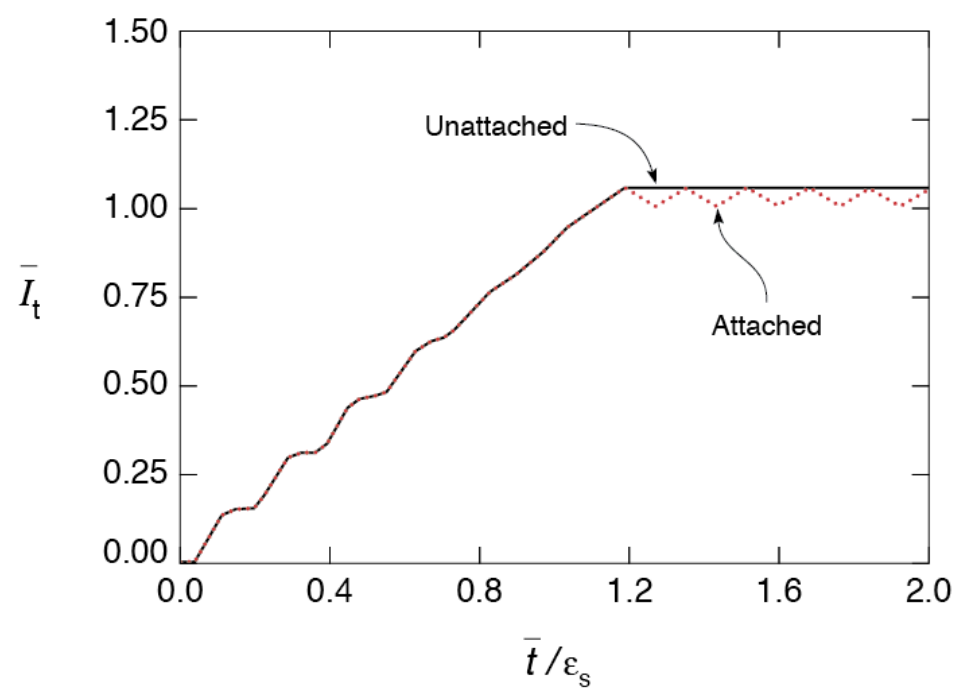

(b)

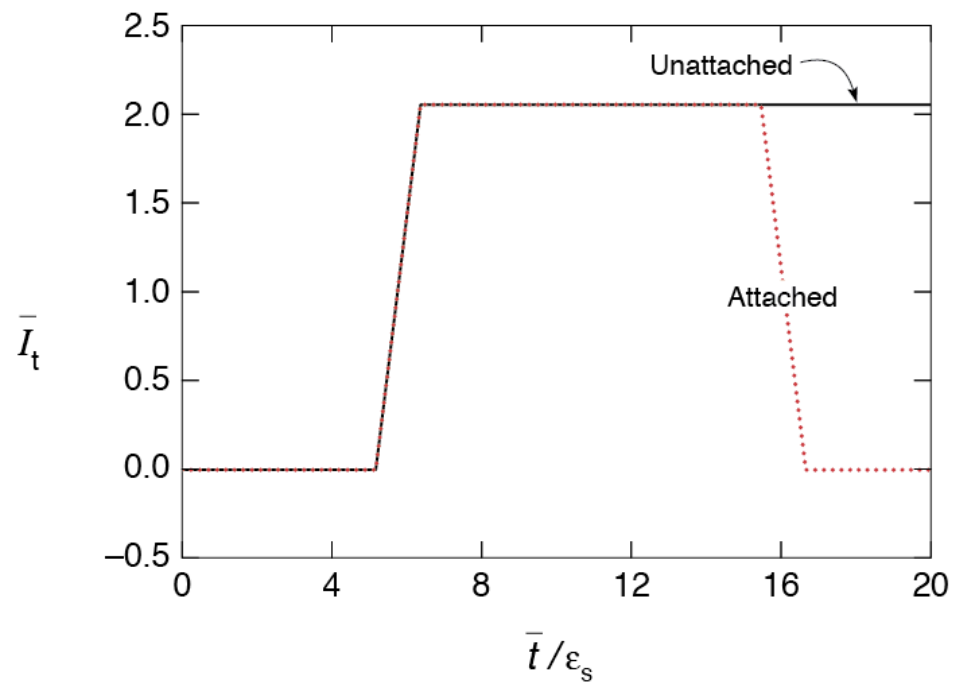

Fig. 10: Discrete/continuum simulation predictions of the time evolution of the normalised transmitted momentum $\bar{I}_{t}$ for the attached and unattached elastic block with (a) $c_{b} / H=0.4$ and (b) $c_{b} / H=50$ for the normalised sand slug velocity $v_{o} / \sqrt{E / \rho_{m}}=0.08$. 


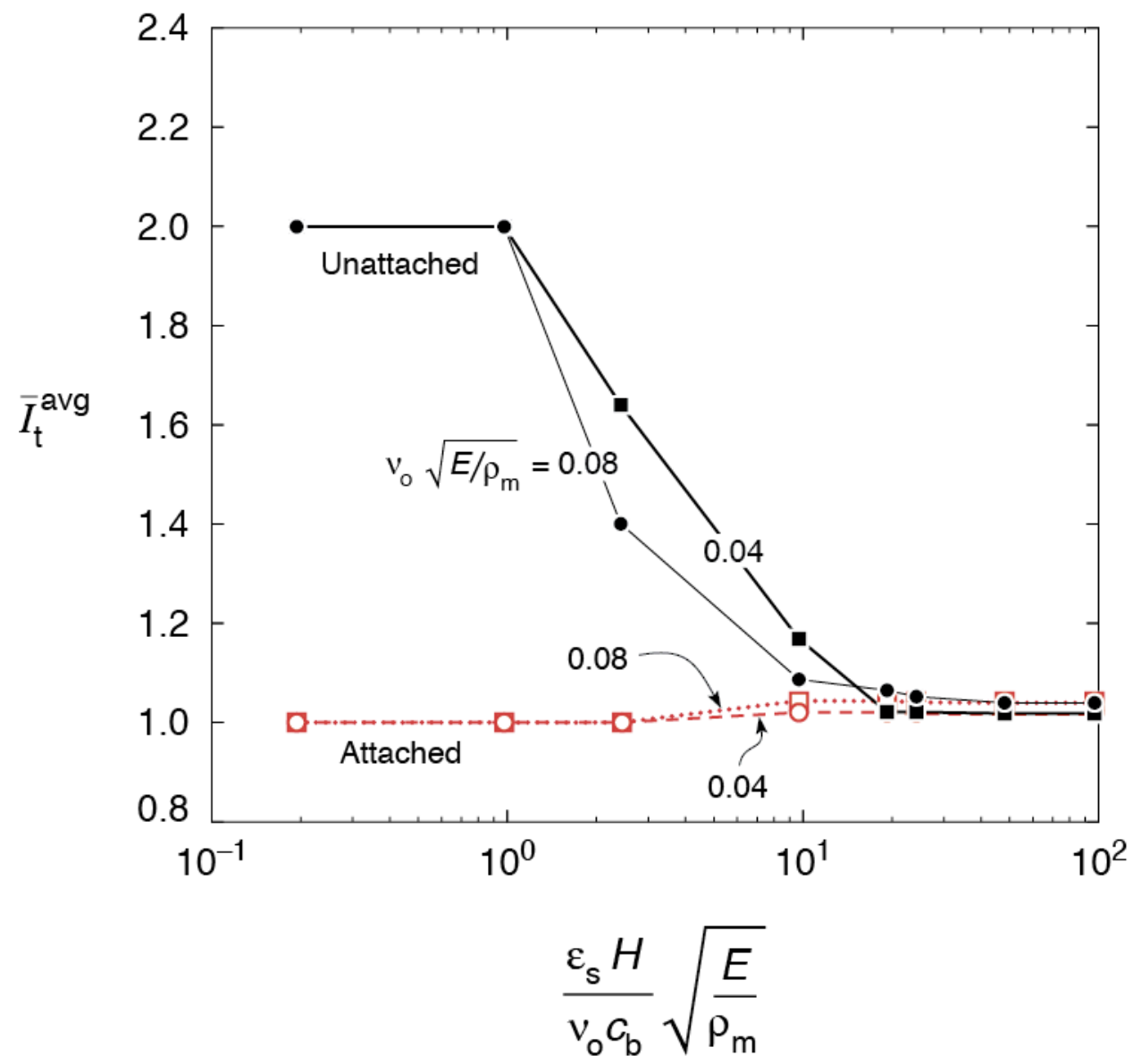

Fig. 11: Discrete/continuum simulation predictions of the normalised average transmitted momentum $I_{t}^{a v g} / I_{o}$ as a function of the time-scale ratio $\bar{\tau}$ for the attached and unattached elastic block. Results are presented for two values of the non-dimensional sand slug velocity $v_{o} / \sqrt{E / \rho_{m}}$. 
(a)

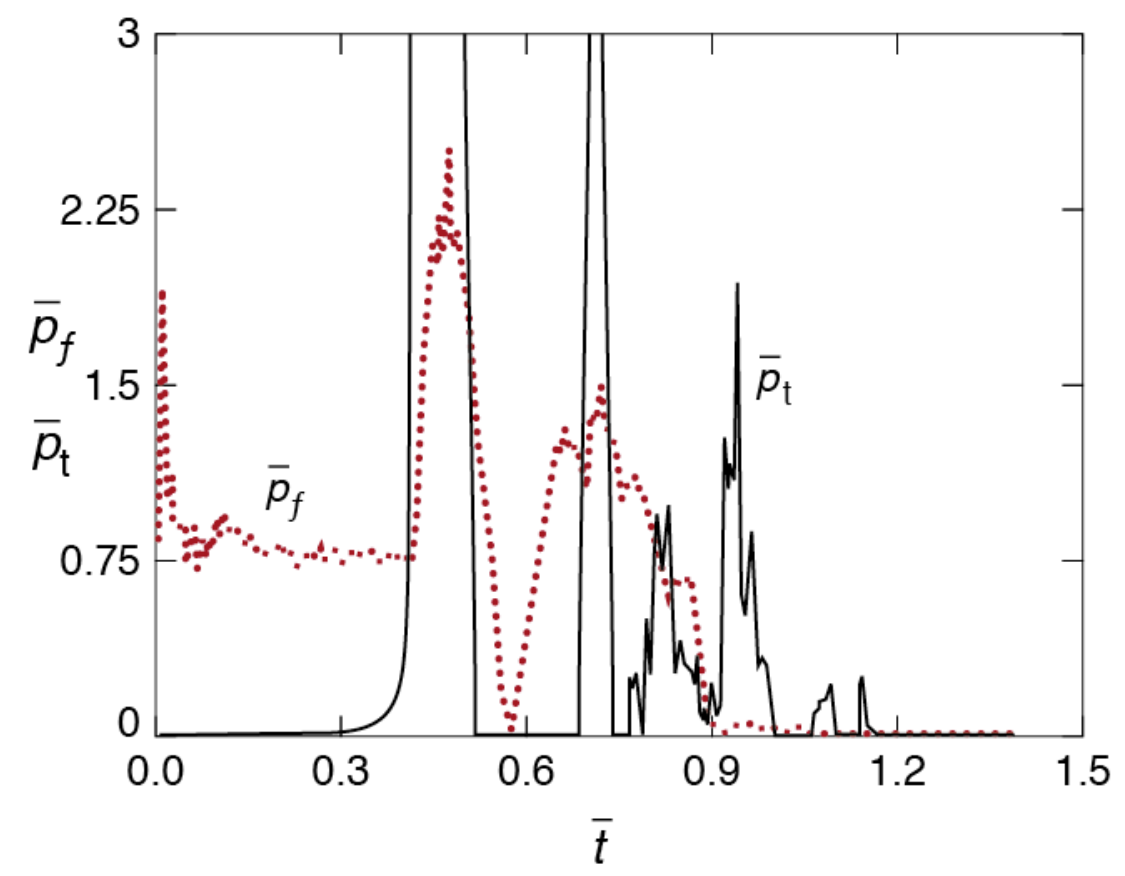

(b)

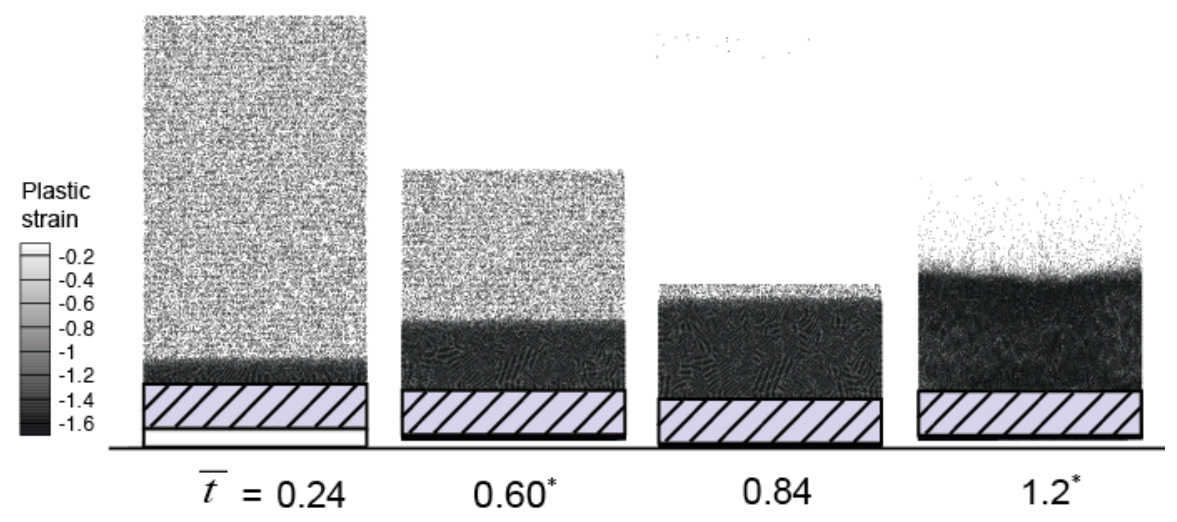

Fig. 12: Discrete/continuum simulation predictions for the unattached buffer plate G-I. (a) Time evolution of the normalised front and transmitted pressures $\bar{p}_{f}$ and $\bar{p}_{t}$, respectively. (b) Snapshots showing the deformation of the buffer plate and sand slug at selected normalised times $\bar{t}$. The ${ }^{*}$ symbol indicates loss of contact between the buffer plate and foundation while the buffer plate face sheets are shown with hatch marks. 
(a)

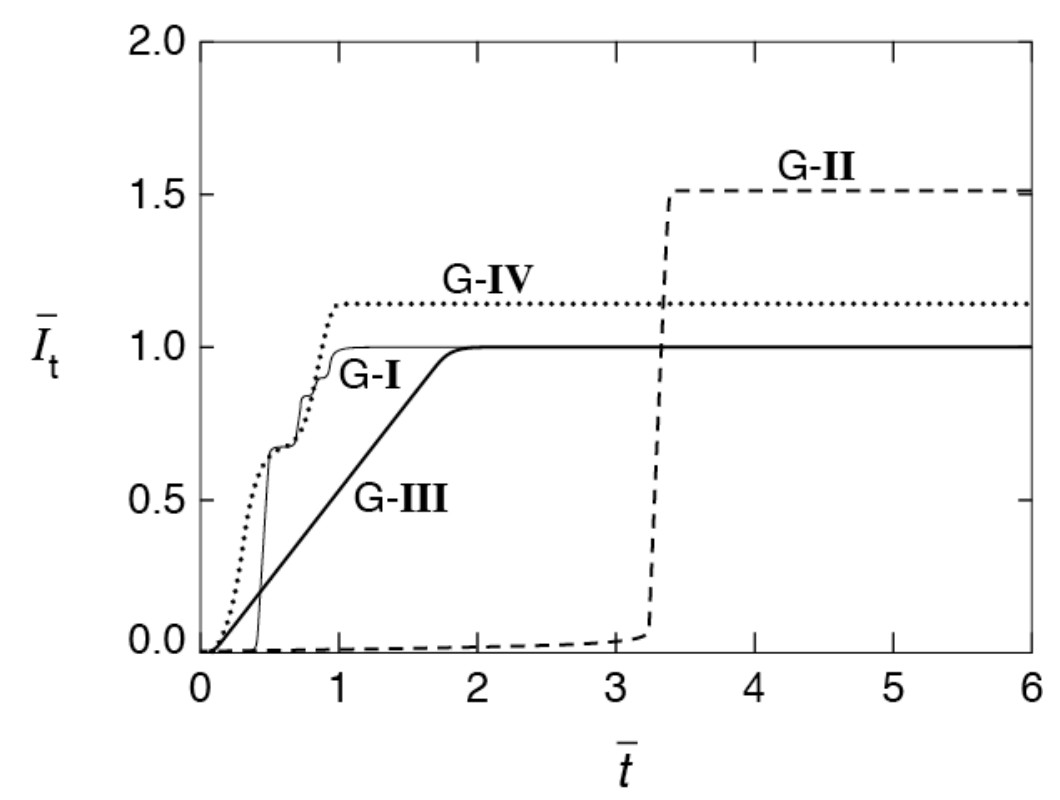

(b)

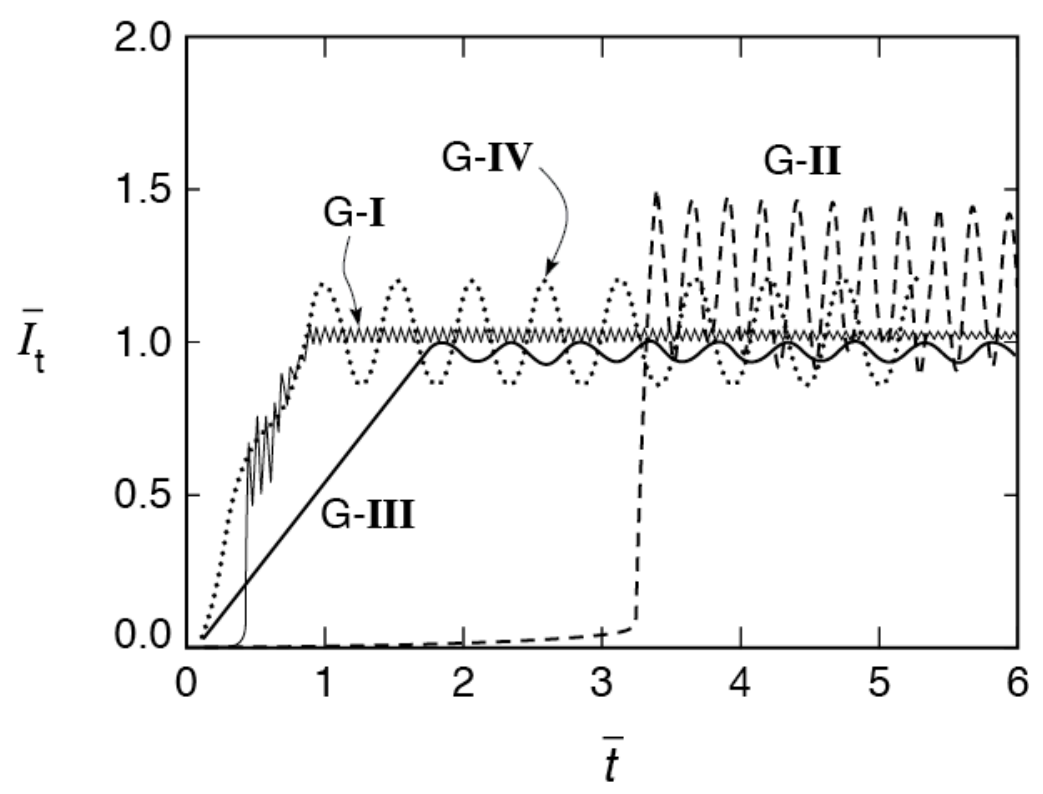

Fig. 13: Time evolution of the normalised transmitted momentum $\bar{I}_{t}$ for the (a) unattached and (b) attached buffer plate geometries G-I through G-IV. 
(a)

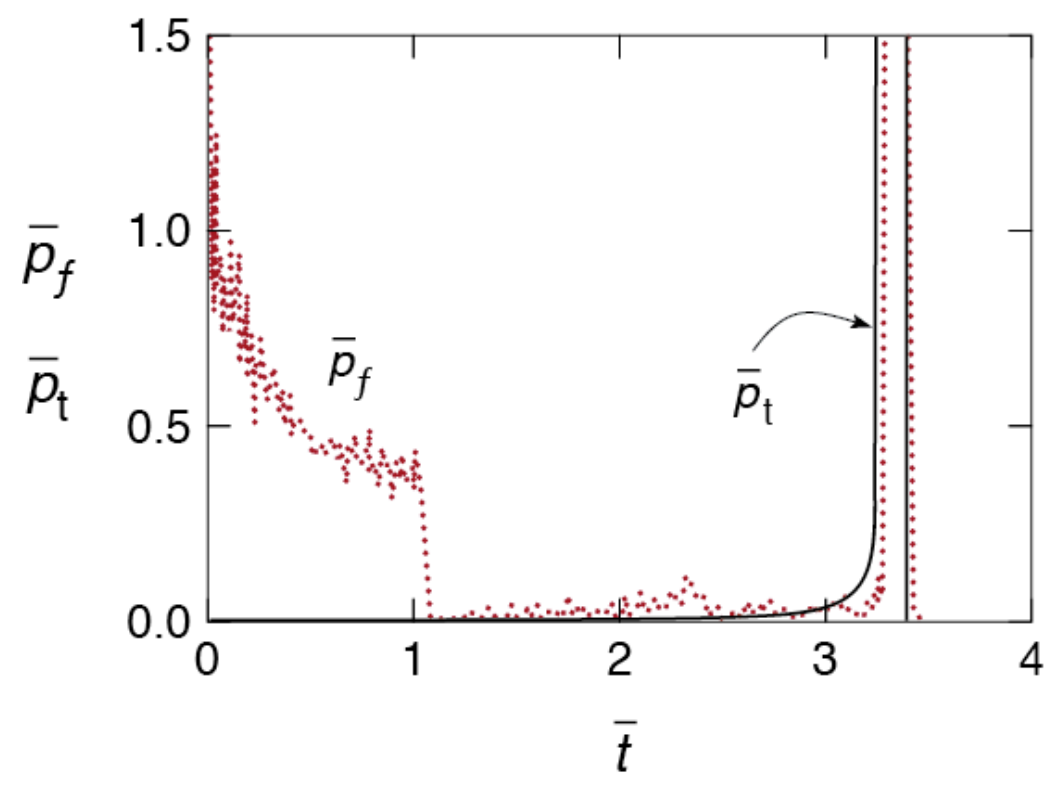

(b)

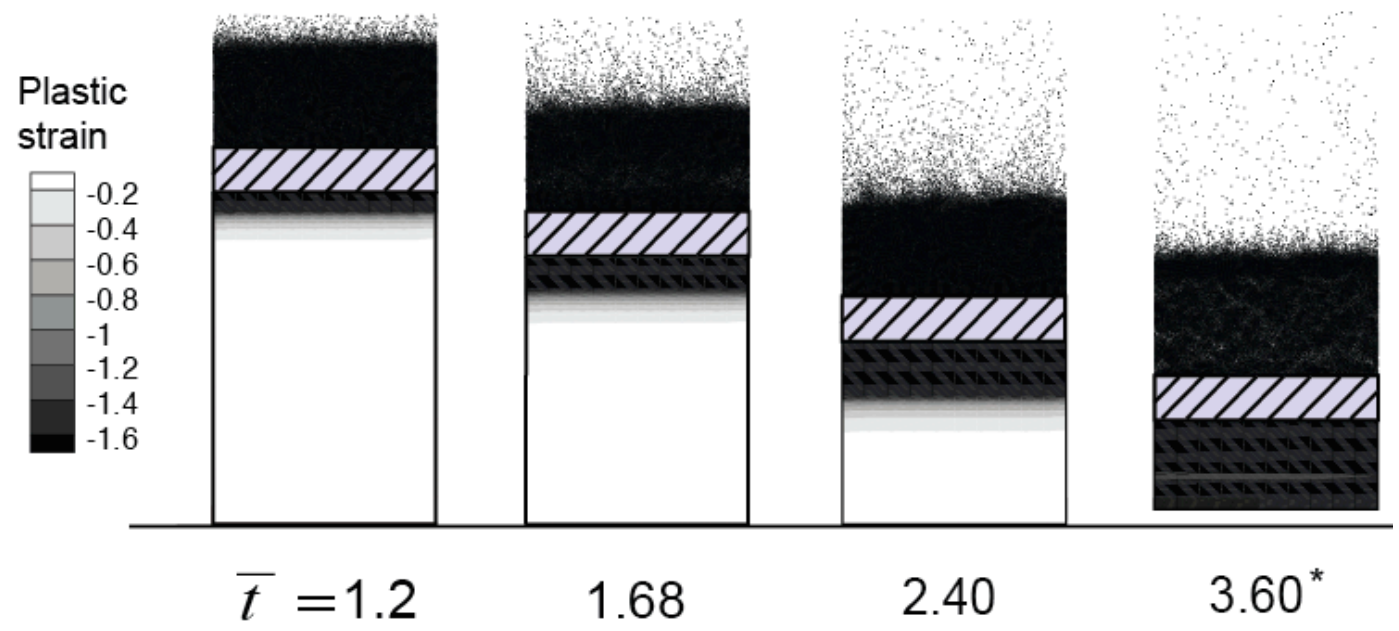

Fig. 14: Discrete/continuum simulation predictions for the unattached buffer plate G-II. (a) Time evolution of the normalised front and transmitted pressures $\bar{p}_{f}$ and $\bar{p}_{t}$, respectively. (b) Snapshots showing the deformation of the buffer plate and sand slug at selected normalised times $\bar{t}$. The ${ }^{*}$ symbol indicates loss of contact between the buffer plate and foundation while the buffer plate face sheets are shown with hatch marks. 
(a)

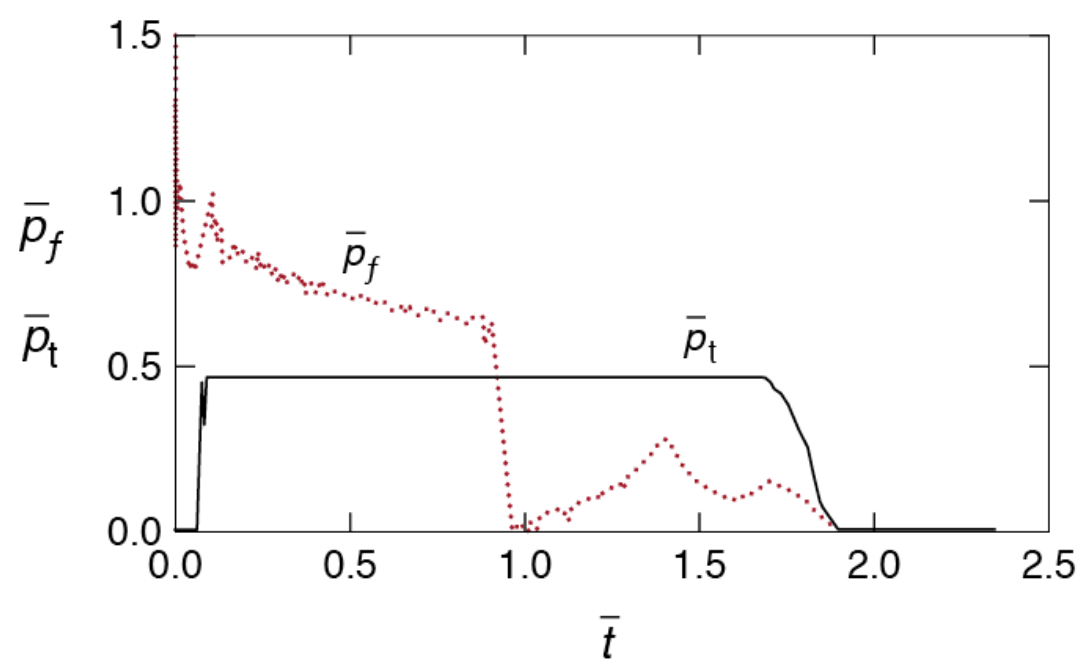

(b)

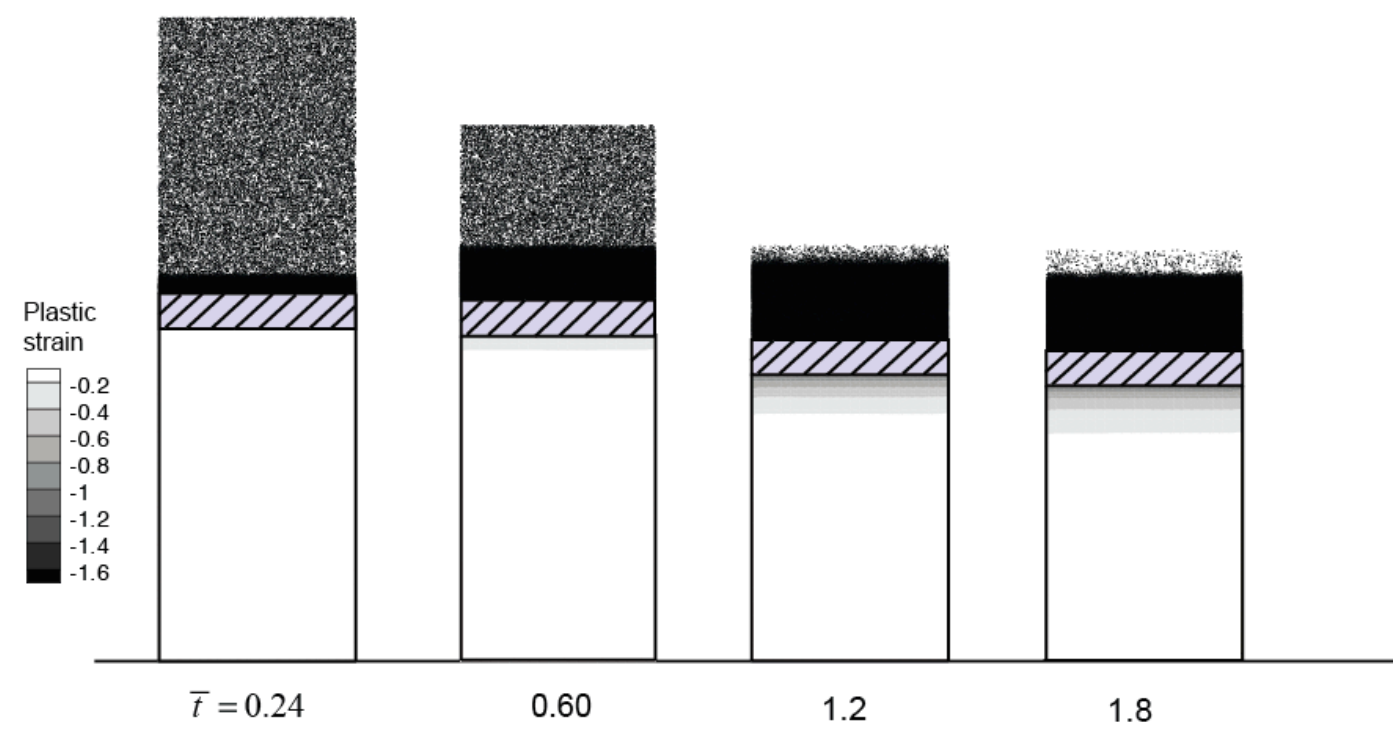

Fig. 15: Discrete/continuum simulation predictions for the unattached buffer plate G-III. (a) Time evolution of the normalised front and transmitted pressures $\bar{p}_{f}$ and $\bar{p}_{t}$, respectively. (b) Snapshots showing the deformation of the buffer plate and sand slug at selected normalised times $\bar{t}$. The ${ }^{*}$ symbol indicates loss of contact between the buffer plate and foundation while the buffer plate face sheets are shown with hatch marks. 
(a)

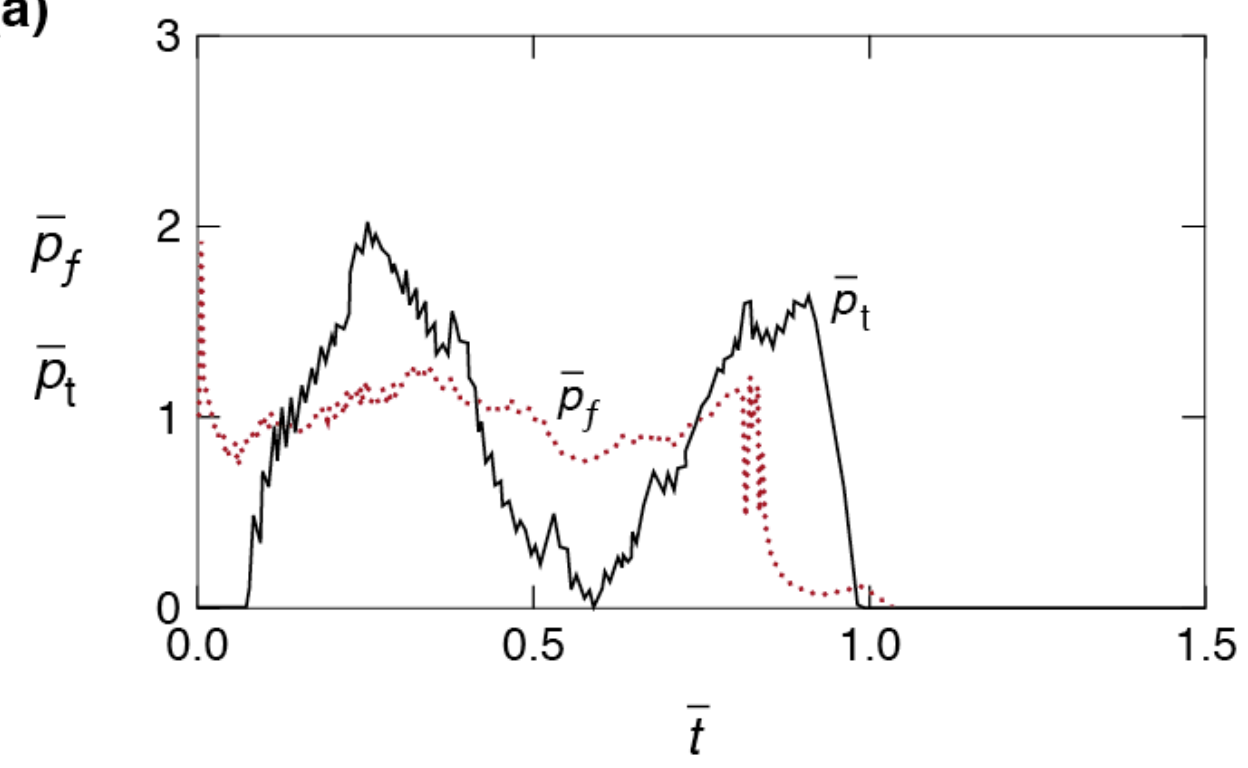

(b)

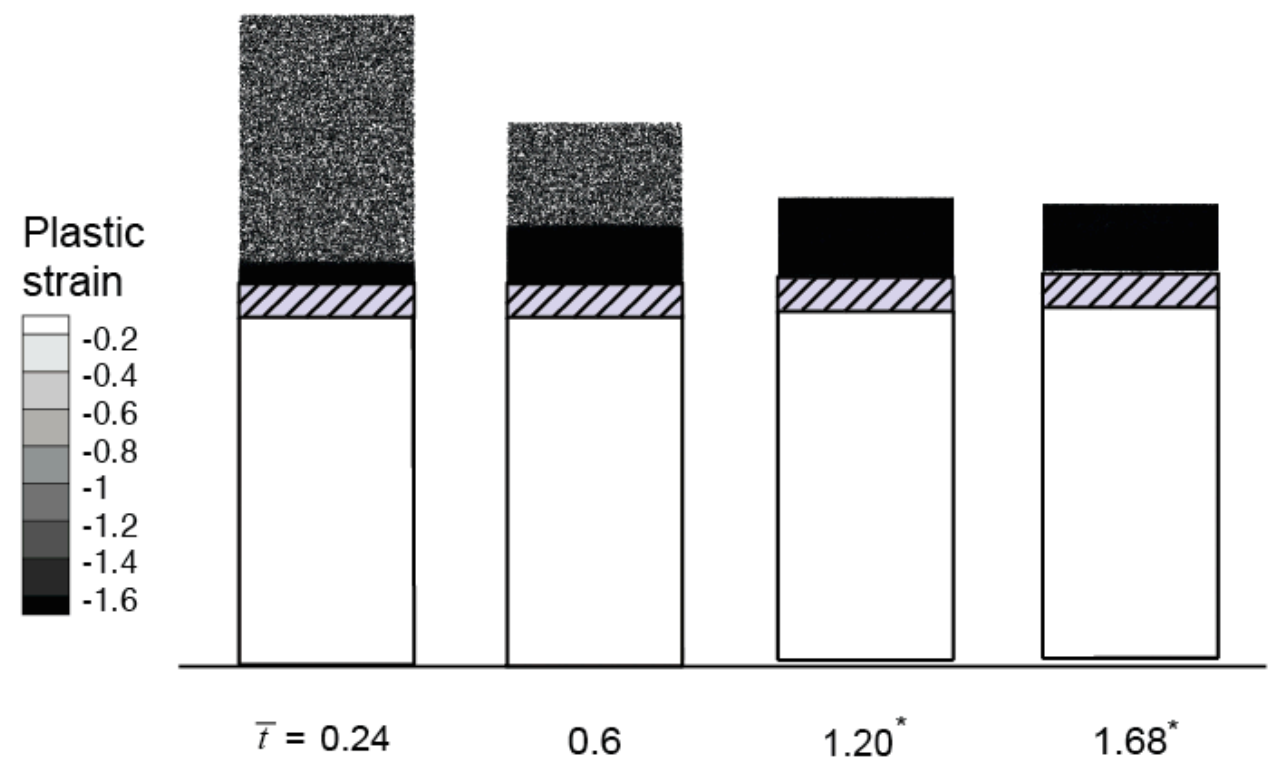

Fig. 16: Discrete/continuum simulation predictions for the unattached buffer plate G-IV. (a) Time evolution of the normalised front and transmitted pressures $\bar{p}_{f}$ and $\bar{p}_{t}$, respectively. (b) Snapshots showing the deformation of the buffer plate and sand slug at selected normalised times $\bar{t}$. The * symbol indicates loss of contact between the buffer plate and foundation while the buffer plate face sheets are shown with hatch marks. 
(a)

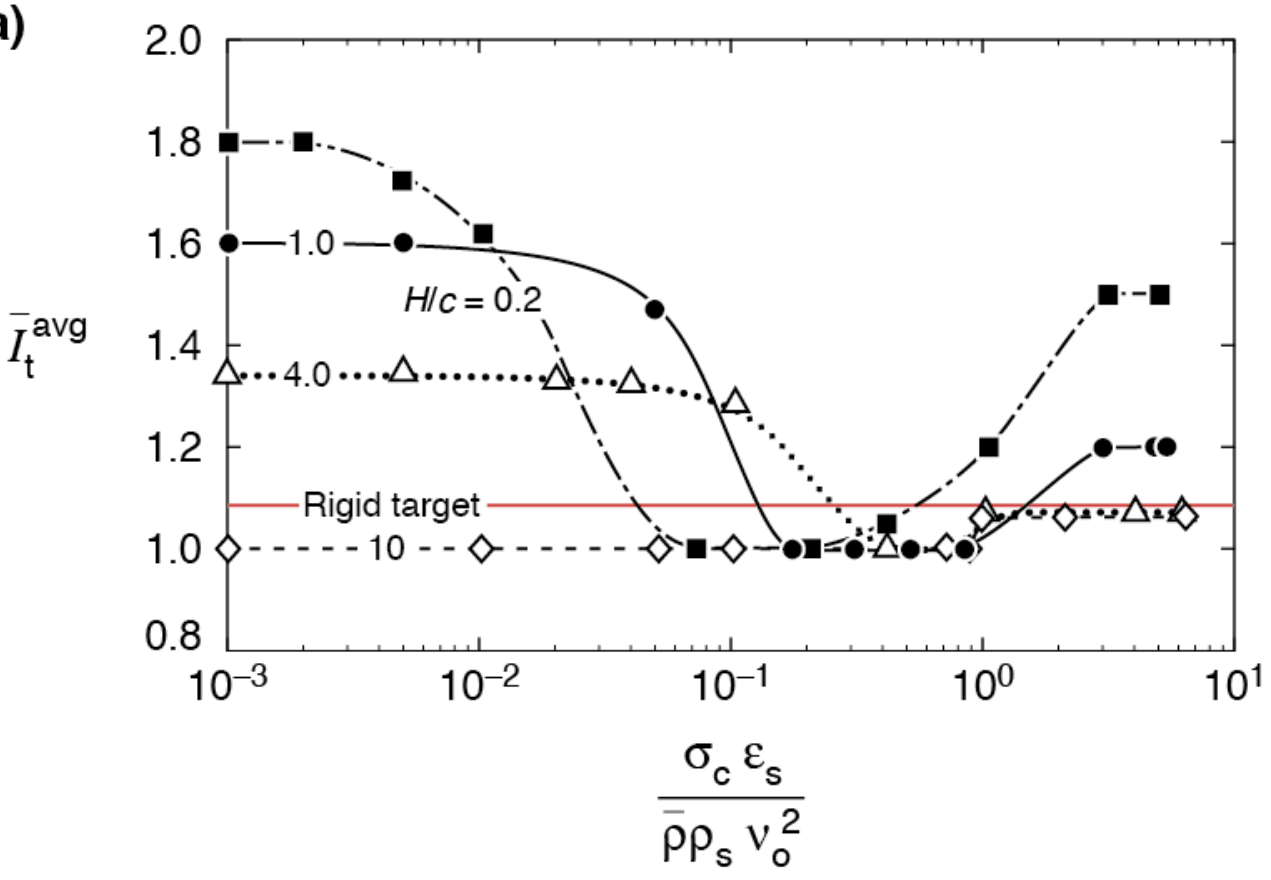

(b)

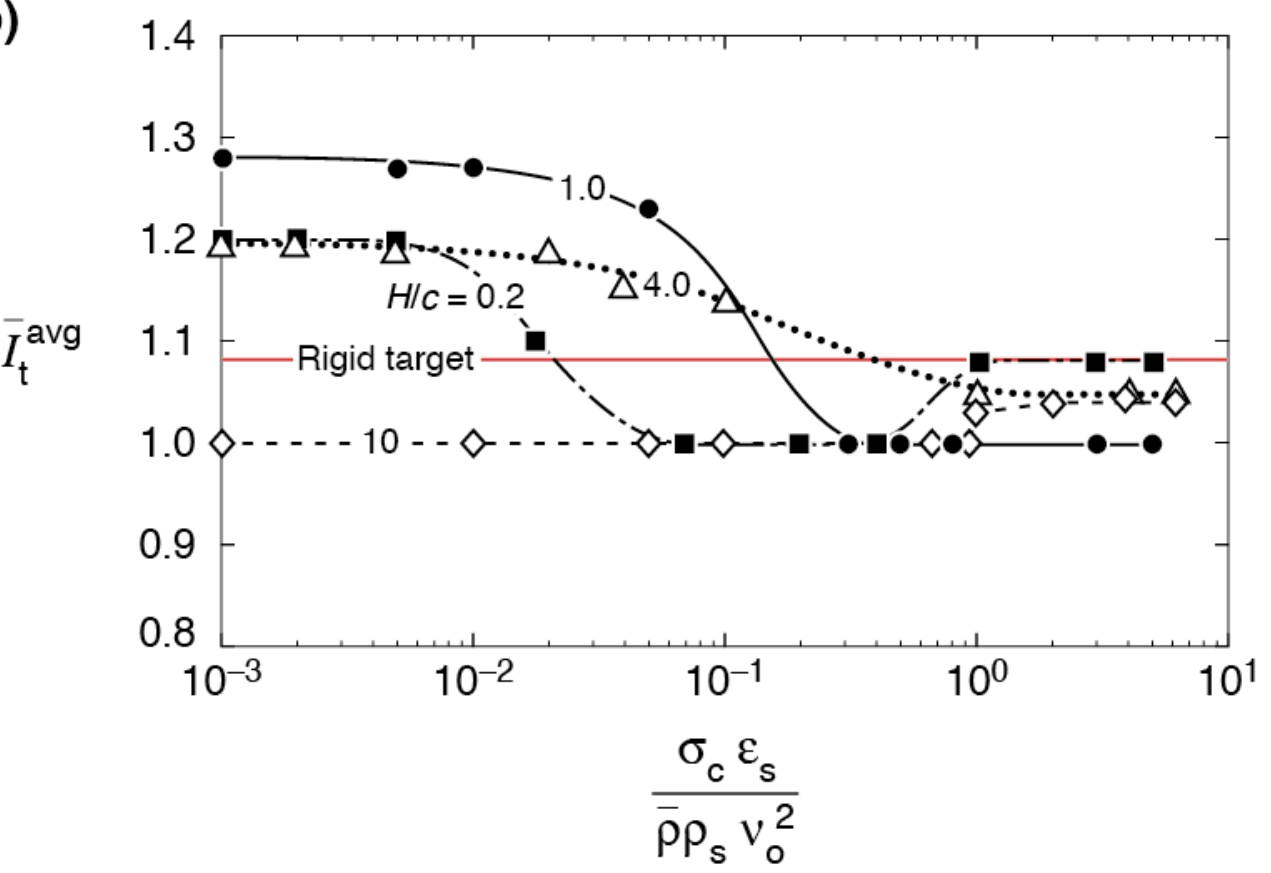

Fig. 17: Discrete/continuum simulation predictions of the normalised average transmitted momentum $I_{t}^{a v g} / I_{0}$ as function of the normalised core strength $\bar{\sigma}_{c}$ for selected values of $\bar{H}$ with $\bar{m}=1, \bar{\rho}=0.2$ and $\psi=0.7$, i.e. case considered in Fig. 5a. The unattached and attached cases are shown in (a) and (b) 